\title{
Conjugacy equivalence relation on subgroups
}

by

\author{
Alessandro Andretta (Torino), Riccardo Camerlo (Torino) \\ and Greg Hjorth (Los Angeles, CA)
}

\begin{abstract}
If $G$ is a countable group containing a copy of $\mathbf{F}_{2}$ then the conjugacy equivalence relation on subgroups of $G$ attains the maximal possible complexity.
\end{abstract}

1. Introduction. This paper is primarily directed towards the study of subgroups of countable groups and the complexity of the conjugacy equivalence relation, $E_{\mathrm{c}}(G, \operatorname{Sgr}(G))$, on the space $\operatorname{Sgr}(G)$ of these subgroups.

It had previously been shown that:

Lemma 1.1 (Stuck, Zimmer, see [8], 3.9). The conjugacy equivalence relation on subgroups of $\mathbf{F}_{2}$, the free group on two generators, is not smooth.

We give the precise definition of smooth below in $\S 2$. Roughly speaking, 1.1 states that there is no Borel function assigning elements of $\mathbb{R}$ as complete invariants to the equivalence classes of $E_{\mathrm{c}}\left(\mathbf{F}_{2}, \operatorname{Sgr}\left(\mathbf{F}_{2}\right)\right)$.

This was recently strengthened in [9]:

Theorem 1.2 (Thomas, Veličković). $E_{\mathrm{c}}\left(\mathbf{F}_{2}, \operatorname{Sgr}\left(\mathbf{F}_{2}\right)\right)$ is a universal countable Borel equivalence relation.

Again the exact definitions can be found in $\S 2$. In essence, 1.2 states that $E_{\mathrm{c}}\left(\mathbf{F}_{2}, \operatorname{Sgr}\left(\mathbf{F}_{2}\right)\right)$ attains the maximal possible complexity. In [3] $\mathrm{Su}$ Gao showed $E_{\mathrm{c}}(G, \operatorname{Sgr}(G))$ to be universal for a variety of finitely generated groups, including $\mathbf{F}_{2}$, all of which contained $\mathbf{F}_{2}$ as a subgroup.

In this present paper we obtain a completely general result:

2000 Mathematics Subject Classification: 03E15, 20E05, 20F05.

Key words and phrases: countable Borel equivalence relations, subgroup conjugacy.

The first author would like to thank the UCLA Math department for hospitality and support during the 1998-1999 academic year, when the results were obtained. The third author gratefully acknowledges support of grant DMS-9970403 and a generous fellowship from the Sloan foundation. 
THEOREM 1.3. For $G$ a countable group containing $\mathbf{F}_{2}$ as a subgroup, $E_{\mathrm{c}}(G, \operatorname{Sgr}(G))$ is universal.

For instance, Theorem 1.3 implies that the conjugacy equivalence relation on $\mathrm{SL}_{2}(\mathbb{Z})$ is universal since, as noted in $[10], \mathrm{SL}_{2}(\mathbb{Z})$ contains a copy of $\mathbf{F}_{2}$. The universality of this specific conjugacy relation does not seem to follow from the arguments of Gao or Thomas and Veličković.

The proof of 1.3 came about as a result of thinking about the following still open and seemingly difficult problem.

QUESTION 1.4. Must any countable Borel equivalence relation including a universal countable Borel equivalence relation itself be universal?

It is easily seen that $E_{\mathrm{c}}\left(\mathbf{F}_{2}, \operatorname{Sgr}\left(\mathbf{F}_{2}\right)\right)$ includes a universal countable Borel equivalence relation, and hence so too does $E_{\mathrm{c}}(G, \operatorname{Sgr}(G))$ whenever $\mathbf{F}_{2} \subseteq G$. The proof of 1.3 below demonstrates universality of $E_{\mathrm{c}}(G, \operatorname{Sgr}(G))$ by going to a subspace of $\operatorname{Sgr}(G)$ on which the universal equivalence relation included in $E_{\mathrm{c}}(G, \operatorname{Sgr}(G))$ exactly equals $E_{\mathrm{c}}(G, \operatorname{Sgr}(G))$; in other words, we restrict to a subspace on which the extra parts of $E_{\mathrm{c}}(G, \operatorname{Sgr}(G))$ are killed off and only the universal countable equivalence relation remains. In some sense the proof can be thought of as a kind of trial effort to affirmatively answer the above open question, but using all the extra information given by the specific context.

The proof of 1.3 also uses the following improvement of a theorem by Dougherty and Kechris:

THEOREM 1.5. For $G$ a countable group of permutations including the recursive permutations, $\cong_{G}^{5}$ is universal.

Here $\simeq_{G}^{5}$ is the equivalence relation on $5^{\mathbb{N}}$ arising from the natural shift action on this space.

Previously it was shown:

Theorem 1.6 (Dougherty, Kechris, see [2]). For $G$ a countable group of permutations including the recursive permutations, $\cong_{G}^{\mathbb{N}}$ is universal.

These results came as an attempt to settle the following open problem.

Question 1.7. Is $\equiv_{\mathrm{T}}$, the relation of Turing equivalence on $\mathbb{N}^{\mathbb{N}}$ (or equivalently $2^{\mathbb{N}}$ ), universal among countable Borel equivalence relations?

An affirmative answer to 1.4 together with 1.6 would yield an affirmative answer to 1.7 , which in turn would refute in a very strong way a conjecture of Martin's in recursion theory - see the Appendix in [6].

2. Notation and definitions. We give a stick figure sketch of the most of the definitions and notation. The reader is referred to [5] for basic facts 
on descriptive set theory, while more extensive introductions to the theory of Borel equivalence relations can be found in [1] or [4].

A topological space $X$ is said to be Polish if it is separable and completely metrizable. For example, $k^{\mathbb{N}}$ and $\mathbb{N}^{\mathbb{N}}$ are both Polish with the topology of pointwise convergence. (Here and below we adopt the usual convention of identifying an integer $k$ with the set $\{0, \ldots, k-1\}$ of all of its predecessors and $A^{B}$ with the set of all functions from $B$ to $A$.) We say that $B \subseteq X$ is Borel if it appears in the smallest $\sigma$-algebra containing the open sets. A function $f: X \rightarrow Y$ between Polish spaces is Borel if $f^{-1}[U]$ is Borel for any open $U \subseteq Y$.

An equivalence relation $E$ on $X$ is Borel if is Borel as a subset of $X \times X$. For $E$ and $F$ Borel equivalence relations on Polish spaces $X$ and $Y$ we say that $E$ is Borel reducible to $F$, written $E \leq_{\mathrm{B}} F$, if there is a Borel function $f: X \rightarrow Y$ which embeds $E$ in $F$, in the sense that for all $x_{1}, x_{2} \in X$,

$$
x_{1} E x_{2} \Leftrightarrow f\left(x_{1}\right) F f\left(x_{2}\right) .
$$

We say that $E$ on $X$ is smooth if $E \leq_{\mathrm{B}} \Delta(\mathbb{R})$, the equality equivalence relation on $\mathbb{R}$. We say that $E$ on $X$ is countable if all of its equivalence classes are countable. A countable Borel equivalence relation $E$ is said to be universal if for any other countable Borel equivalence relation $F$ we have $F \leq_{\mathrm{B}} E$.

It is a somewhat non-trivial fact that there is a universal countable Borel equivalence relation. We work towards giving an example due to Dougherty, Jackson, and Kechris.

Let $G$ be an infinite countable group. Then $G$ acts on $2^{G}$ by left shift, namely we define $G \times 2^{G} \rightarrow 2^{G},(g, x) \mapsto g . x$, to be

$$
\forall h \in G\left((g . x)(h)=x\left(g^{-1} h\right)\right) .
$$

We then denote the resulting orbit equivalence relation on $2^{G}$ by $E(G, 2)$. Note that $2^{G}$ is Polish, being homeomorphic to $2^{\mathbb{N}}$, and that $E(G, 2)$ is countable Borel.

Theorem 2.1 (Dougherty, Jackson, Kechris, see [1]). E(F $\left.\mathbf{F}_{2}, 2\right)$ is universal.

For $G$ a countable group, we let $\operatorname{Sgr}(G)$ be the collection of all subgroups of $G$. Under the natural identification of $\mathcal{P}(G)$ with $2^{G}$ we find that $\operatorname{Sgr}(G)$ is a closed subset of $2^{G}$, and hence a Polish space in its own right. We then let $G$ act on $\operatorname{Sgr}(G)$ by conjugation and define $E_{\mathrm{c}}(G, \operatorname{Sgr}(G))$ to be the resulting orbit equivalence relation. Thus for $H_{1}, H_{2} \subseteq G$ we have

$$
H_{1} E_{\mathrm{c}}(G, \operatorname{Sgr}(G)) H_{2} \Leftrightarrow \exists g \in G\left(g H_{1} g^{-1}=H_{2}\right) .
$$

If $X$ is a set then $\operatorname{Sym}(X)$ is the group of all permutations of $X$. Usually, $\operatorname{Sym}(\mathbb{N})$ is denoted by $S_{\infty}$. We let $S_{\infty}$ act on the right on $k^{\mathbb{N}}$, by $k^{\mathbb{N}} \times$ 
$S_{\infty} \rightarrow k^{\mathbb{N}},(x, g) \mapsto x \circ g$. We then obtain for any subgroup $G \subseteq S_{\infty}$ the corresponding equivalence relation $\cong_{G}^{k}$ :

$$
x \cong_{G}^{k} y \Leftrightarrow \exists g \in G(x \circ g=y) .
$$

Similarly we define $\cong{ }_{G}^{\mathbb{N}}$ on $\mathbb{N}^{\mathbb{N}}$.

3. Groups of permutations. We now prove Theorem 1.5. In fact, we will prove a slightly stronger assertion.

THEOREM 3.1. There is a countable group $G_{0} \subseteq S_{\infty}$ of recursive permutations such that $G_{0} \cong \mathbf{F}_{2}$, and for every countable group $G$ with $G_{0} \subseteq G \subseteq$ $S_{\infty}$, the equivalence relation $\cong_{G}^{5}$ on $5^{\mathbb{N}}$ is universal among countable Borel equivalence relations. In particular, this holds when $G=\operatorname{Rec}$, the group of recursive bijections.

Proof. Rather than working with subgroups of $S_{\infty}$ we will look at subgroups $G$ of $\operatorname{Sym}(M)$, where $M=\mathbf{F}_{2} \times \mathbb{N}^{2}$. The set $M$ is best visualized as $\mathbf{F}_{2}$-many copies of the square $\mathbb{N} \times \mathbb{N}$ :

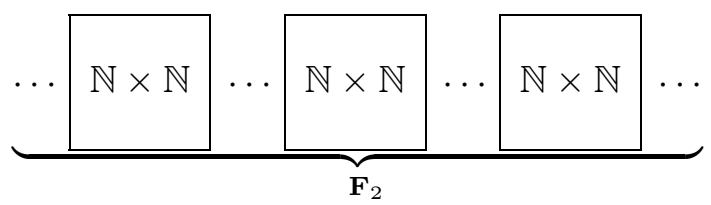

A generic element of $M$ is denoted by $(w, n, k)$ with $n$ ranging on the $x$-axis and $k$ ranging on the $y$-axis of the $w$ th square. Thus a set of the form $\left\{(w, n, k) \in M \mid k_{0} \leq k \leq k_{1}\right\}$ is called a horizontal strip of $M$.

For every $w \in \mathbf{F}_{2}$ let $\widehat{w} \in \operatorname{Sym}(M)$ be defined by

$$
\widehat{w}(v, n, k)=(v w, n, k)
$$

and let $G_{0}=\left\{\widehat{w} \mid w \in \mathbf{F}_{2}\right\}$. (Note that the map $\mathbf{F}_{2} \rightarrow G_{0}, w \mapsto \widehat{w^{-1}}$, is an isomorphism.) An element of the form $\widehat{w}$ acts by right multiplication on the $\mathbf{F}_{2}$-coordinate, leaving the other two coordinates unchanged. Let $G$ be a countable group with $G_{0} \subseteq G \subseteq \operatorname{Sym}(M)$. If $G$ were equal to $G_{0}$ then we would be done by 2.1 and $E\left(G_{0}, 2\right) \leq_{\mathrm{B}} \cong{ }_{G_{0}}^{5}$. So we try to analyze how different from an element of $G_{0}$ can an element of $G$ be. Fix an enumeration $\left(g_{i}\right)_{i>0}$ of $G$. (For notational reasons it is more convenient to avoid 0 as an index here.) An element $g \in G$ is called a quasi-shift above $l$ iff $\forall(w, n, k) \in M$

$$
\left[k>l \Rightarrow g(w, n, k)=(\varphi(w, n, k), n, k) \& g^{-1}(w, n, k)=(\psi(w, n, k), n, k)\right]
$$

for some functions $\varphi, \psi: M \rightarrow \mathbf{F}_{2}$. In other words, a quasi-shift respects pointwise the second and third coordinates of $M$. If the functions $\varphi, \psi$ above depend on $\mathbf{F}_{2}$ only, i.e., $g(w, n, k)=(\varphi(w), n, k)$ and $g^{-1}(w, n, k)=$ $(\psi(w), n, k)$, then $g$ is a shift. Elements of $G_{0}$ are particular types of shifts. 
An increasing sequence $\left(l_{i}\right)_{i \in \mathbb{N}}$ of integers is defined inductively.

Set $l_{0}=-1$ and suppose $l_{i-1}$ has been defined for some $i \geq 1$. We now distinguish two cases:

- $g_{i}$ is not a quasi-shift above $l_{i-1}$, i.e., there is a $w_{0} \in \mathbf{F}_{2}$ and there are $\left(n_{0}, k_{0}\right) \in \mathbb{N}^{2}$ such that $g_{i}\left(w_{0}, n_{0}, k_{0}\right)=\left(w_{0}^{\prime}, n_{0}^{\prime}, k_{0}^{\prime}\right)$ and $\left(n_{0}, k_{0}\right) \neq\left(n_{0}^{\prime}, k_{0}^{\prime}\right)$, with $k_{0}>l_{i-1}$ or $k_{0}^{\prime}>l_{i-1}$. Then we have the following possibilities:

(i) $g_{i}\left(w_{0}, n_{0}, k_{0}\right)=\left(w_{0}^{\prime}, n_{0}^{\prime}, k_{0}^{\prime}\right)$ for some $\left(w_{0}, n_{0}, k_{0}\right),\left(w_{0}^{\prime}, n_{0}^{\prime}, k_{0}^{\prime}\right) \in M$ with $k_{0}>l_{i-1}$ and $k_{0}>k_{0}^{\prime}$;

(ii) $g_{i}^{-1}\left(w_{0}, n_{0}, k_{0}\right)=\left(w_{0}^{\prime}, n_{0}^{\prime}, k_{0}^{\prime}\right)$ for some $\left(w_{0}, n_{0}, k_{0}\right),\left(w_{0}^{\prime}, n_{0}^{\prime}, k_{0}^{\prime}\right) \in M$ with $k_{0}>l_{i-1}$ and $k_{0}>k_{0}^{\prime}$, and (i) fails;

(iii) $g_{i}\left(w_{0}, n_{0}, k_{0}\right)=\left(w_{0}^{\prime}, n_{0}^{\prime}, k_{0}\right)$ for some $\left(w_{0}, n_{0}, k_{0}\right),\left(w_{0}^{\prime}, n_{0}^{\prime}, k_{0}\right) \in M$ with $k_{0}>l_{i-1}$ and $n_{0} \neq n_{0}^{\prime}$, and both (i) and (ii) fail.

Then set $l_{i}=k_{0}$, for the least possible $k_{0}$ as above.

- Otherwise, that is: $g_{i}$ is a quasi-shift above $l_{i-1}$. Then set $l_{i}=l_{i-1}+1$.

Suppose $g_{i}$ is a quasi-shift above $l_{i-1}$. Then $g_{i}$ shuffles the $l_{i}$ th rows among the various squares, and, in particular, $\forall n \in \mathbb{N} \exists w \in \mathbf{F}_{2}\left(g_{i}\left(1_{\mathbf{F}_{2}}, n, l_{i}\right)\right.$ $\left.=\left(w, n, l_{i}\right)\right)$.

We say that $g_{i}$ is good if there is an increasing sequence $\left(n_{m}\right)_{m}$ of natural numbers and a fixed $w_{\infty} \in \mathbf{F}_{2}$ such that

$$
\forall m \in \mathbb{N}\left(g_{i}\left(1_{\mathbf{F}_{2}}, n_{m}, l_{i}\right)=\left(w_{\infty}, n_{m}, l_{i}\right)\right) .
$$

If $g_{i}$ is not good then it is called bad. In this case we can find an increasing sequence $\left(n_{m}\right)_{m}$ of natural numbers and a sequence $\left(w_{m}\right)_{m}$ of distinct elements in $\mathbf{F}_{2}$ such that

$$
\forall m \in \mathbb{N}\left(g_{i}\left(1_{\mathbf{F}_{2}}, n_{m}, l_{i}\right)=\left(w_{m}, n_{m}, l_{i}\right)\right) .
$$

Let also $z_{m+1}=w_{m+1} w_{m}^{-1}$ and define $\mu: 2^{\mathbf{F}_{2}} \rightarrow\{1,2, \ldots, \infty\}$ by

$$
\mu(x)=\left\{\begin{array}{l}
\text { the least } m \geq 1 \text { such that } z_{m} . x \neq x, \\
\infty \quad \text { if } \forall m \geq 1\left(z_{m} . x=x\right) .
\end{array}\right.
$$

We record a few simple facts about $\mu$ whose proof is left to the reader.

Claim 3.2. $\forall j<\mu\left(w_{0} \cdot x\right)\left(w_{j} \cdot x=w_{0} \cdot x\right)$, and if $\mu\left(w_{0} \cdot x\right)<\infty$ then $\mu\left(w_{\mu\left(w_{0} . x\right)} \cdot x\right) \leq \mu\left(w_{0} \cdot x\right)$.

Obviously all of the above $\left(n_{m}\right.$ 's, $w_{m}$ 's, $z_{m}$ 's, $\mu$ ) depend on $i$ so we should really write $n_{m}^{(i)}, w_{m}^{(i)}$, etc.

We shall define a Borel map $2^{\mathbf{F}_{2}} \rightarrow 5^{M}, x \mapsto x^{*}$, witnessing the reduction $E\left(\mathbf{F}_{2}, 2\right) \leq_{\mathrm{B}} \cong_{G}^{5}$. The map $x^{*}: M \rightarrow 5$ assigns to each element of $M$ a colour, i.e., an integer between 0 and 4 . The colouring of $x^{*}$ will encode $x$ in a sufficiently "rigid" way so that for all $x, y \in 2^{\mathbf{F}_{2}}$,

1. $\forall w \in \mathbf{F}_{2}\left(w \cdot x=y \Rightarrow x^{*} \circ \widehat{w}=y^{*}\right)$, hence $x E\left(\mathbf{F}_{2}, 2\right) y \Rightarrow x^{*} \cong_{G}^{5} y^{*}$, 
2. $\forall g \in G \exists w \in \mathbf{F}_{2}\left(x^{*} \circ g=y^{*} \Rightarrow w \cdot x=y\right)$, and hence $x^{*} \cong_{G}^{5} y^{*} \Rightarrow$ $x E\left(\mathbf{F}_{2}, 2\right) y$.

The function $x^{*}: M \rightarrow 5$ will be defined on the horizontal strips

$$
\left\{(w, n, k) \in M \mid l_{i-1}<k \leq l_{i}\right\}
$$

by induction on $i \geq 1$ in such a way that if $g_{i}$ is not a quasi-shift then the value of $x^{*}$ is independent of $x$, i.e.,

$g_{i}$ not a quasi-shift

$$
\Rightarrow \forall x, y \in 2^{\mathbf{F}_{2}} \forall(w, n, k)\left(l_{i-1}<k \leq l_{i} \Rightarrow x^{*}(w, n, k)=y^{*}(w, n, k)\right) .
$$

The horizontal strip $\left\{(w, n, k) \in M \mid l_{i-1}<k \leq l_{i}\right\}$ is used to "kill" the $g_{i}$ 's which are not quasi-shifts, i.e., it is used to make sure that $\forall x, y \in$ $2^{\mathbf{F}_{2}}\left(x^{*} \circ g_{i} \neq y^{*}\right)$. If instead $g_{i}$ is a quasi-shift, then $l_{i-1}+1=l_{i}$ and the set $\left\{\left(w, n, l_{i}\right) \mid n \in \mathbb{N}\right\}$ is used either to kill $g_{i}$ or else to encode $w . x$. Also, the map $x \mapsto\left\{(w, n, k) \mid x^{*}(w, n, k)=4\right\}$ will be constant, that is, whether or not $x^{*}(w, n, k)=4$ will not depend on $x$ but on $(w, n, k)$ only.

Now the details. We will consider two cases depending on whether or not $g_{i}$ is a quasi-shift.

\section{$g_{i}$ is a quasi-shift above $l_{i-1}$.}

Then $l_{i-1}+1=l_{i}$ and we only have to define $x^{*}\left(w, n, l_{i}\right)$ for $w \in \mathbf{F}_{2}$ and $n \in \mathbb{N}$. Let $\left(n_{m}\right)_{m}$ be as in the definition of good/bad quasi-shift. Fix an enumeration without repetitions $\left(u_{m}\right)_{m}$ of $\mathbf{F}_{2}$.

(0) For $n \notin\left\{n_{m} \mid m \in \mathbb{N}\right\}$ set $x^{*}\left(w, n, l_{i}\right)=0$.

(1) If $g_{i}$ is good then set for every $w \in \mathbf{F}_{2}$ and $m \in \mathbb{N}$

$$
x^{*}\left(w, n_{m}, l_{i}\right)=(w \cdot x)\left(u_{m}\right) \in\{0,1\} .
$$

In other words, we are encoding $w \cdot x$ on the sequence $\left(n_{m}\right)_{m}$ in the $w$ th square. Therefore for all $(w, m) \in \mathbf{F}_{2} \times \mathbb{N}$,

$$
x^{*}\left(w, n_{m}, l_{i}\right)=(w \cdot x)\left(u_{m}\right)=(w \cdot x)^{*}\left(1_{\mathbf{F}_{2}}, n_{m}, l_{i}\right)
$$

and thus $\forall w \in \mathbf{F}_{2} \forall n \in \mathbb{N}\left(x^{*}\left(w, n, l_{i}\right)=(w \cdot x)^{*}\left(1_{\mathbf{F}_{2}}, n, l_{i}\right)\right)$ by $(0)$.

(2) If $g_{i}$ is bad and $\mu(w \cdot x)=\infty$ then set, for every $m \in \mathbb{N}$,

$$
x^{*}\left(w, n_{0}, l_{i}\right)=0, \quad x^{*}\left(w, n_{1}, l_{i}\right)=3, \quad x^{*}\left(w, n_{m+2}, l_{i}\right)=(w \cdot x)\left(u_{m}\right) .
$$

Also in this case $w \cdot x$ is encoded on $\left(n_{m}\right)_{m}$ in the $w$ th square, but a "flag" 03 is attached at the very beginning. Again it is easy to check that $\forall w \in$ $\mathbf{F}_{2} \forall n \in \mathbb{N}\left(x^{*}\left(w, n, l_{i}\right)=(w \cdot x)^{*}\left(1_{\mathbf{F}_{2}}, n, l_{i}\right)\right)$.

So we may assume $g_{i}$ is bad and $0<\mu(w \cdot x)<\infty$. We will need the following result which is an immediate consequence of Proposition 4.6 of [7]. 
Lemma 3.3. Let $T: X \rightarrow X$ be a Borel transformation on a standard Borel space $X$. There are pairwise disjoint Borel sets $X^{(0)}, X^{(1)}, X^{(2)}$ such that $X^{(0)} \cup X^{(1)} \cup X^{(2)}=X$ and such that for all $x \in X$ and $j \in\{0,1,2\}$,

$$
\left(x \neq T(x) \& x \in X^{(j)}\right) \Rightarrow T(x) \notin X^{(j)} .
$$

That is to say: if $x$ is not a fixed point of $T$ then $x$ and $T(x)$ belong to different pieces of the partition $\left\{X^{(0)}, X^{(1)}, X^{(2)}\right\}$.

For every $m \in \mathbb{N}$ the map $T_{m}: 2^{\mathbf{F}_{2}} \rightarrow 2^{\mathbf{F}_{2}}, x \mapsto z_{m} . x$, is a Borel transformation, where $\left(z_{m}\right)_{m}$ is as in the definition of bad quasi-shift. Let $X_{m}^{(0)}, X_{m}^{(1)}$, and $X_{m}^{(2)}$ be as in the lemma, with $T=T_{m}$. Then set

$$
J_{m}(x)=\text { the unique } j \in\{0,1,2\} \text { such that } x \in X_{m}^{(j)} .
$$

(Note that the definition of $J_{m}$ depends on $z_{m}$ and hence on the index $i$.)

(3) If $g_{i}$ is bad and $0<\mu(w \cdot x)<\infty$ then

$$
x^{*}\left(w, n_{m}, l_{i}\right)= \begin{cases}3 & \text { if } m<\mu(w \cdot x)-1, \\ J_{\mu(w \cdot x)}(w \cdot x) & \text { if } m=\mu(w \cdot x)-1 \text { or } m=\mu(w \cdot x), \\ 3 & \text { if } m>\mu(w \cdot x) .\end{cases}
$$

In this case $x^{*}$ is not encoding $w \cdot x$ on $\left\{\left(w, n_{m}, l_{i}\right) \mid m \in \mathbb{N}\right\}$ : the particular definition of $x^{*}\left(w, n_{m}, l_{i}\right)$ will be used to show that $x^{*} \circ g_{i} \neq y^{*}$, for any $y \in 2^{\mathbf{F}_{2}}$.

We now want to check that the behaviour of $x^{*}$ on the set $\left\{\left(1_{\mathbf{F}_{2}}, n_{m}, l_{i}\right) \mid\right.$ $m \in \mathbb{N}\}$ determines whether we are in case (1), (2), or (3) above.

Claim 3.4. Let $i \geq 1$ be such that $g_{i}$ is a quasi-shift above $l_{i-1}$ and let $x, y \in 2^{\mathbf{F}_{2}}$ be such that $\forall m\left(x^{*}\left(1_{\mathbf{F}_{2}}, n_{m}, l_{i}\right)=y^{*}\left(1_{\mathbf{F}_{2}}, n_{m}, l_{i}\right)\right)$.

Suppose the definition of $x^{*}$ on the set $\left\{\left(1_{\mathbf{F}_{2}}, n_{m}, l_{i}\right) \mid m \in \mathbb{N}\right\}$ is as in case (1), (2), or (3). Then also the definition of $y^{*}$ on $\left\{\left(1_{\mathbf{F}_{2}}, n_{m}, l_{i}\right) \mid m \in \mathbb{N}\right\}$ is as in case (1), (2), or (3), respectively.

Moreover, the sequence $\left(x^{*}\left(1_{\mathbf{F}_{2}}, n_{m}, l_{i}\right)\right)_{m}$ is of the form:

- an element of $2^{\mathbb{N}}$ if $x^{*}$ is defined as in (1),

- 03 followed by an element of $2^{\mathbb{N}}$ if $x^{*}$ is defined as in (2),

- $(3, \ldots, 3, n, n, 3,3, \ldots)$ starting with a possibly empty sequence of 3 's, then two consecutive $n$ 's with $n \in\{0,1,2\}$, at position $\mu(x)-1$ and $\mu(x)$, and then 3 from now on. This happens when $x^{*}$ is defined as in (3).

Proof. If $x^{*}$ on $\left\{\left(1_{\mathbf{F}_{2}}, n_{m}, l_{i}\right) \mid m \in \mathbb{N}\right\}$ is defined as in (1) then $g_{i}$ must be good, hence also $y^{*}$ is defined as in (1).

For the other cases notice that if $x^{*}\left(1_{\mathbf{F}_{2}}, n_{0}, l_{i}\right) \in\{1,2,3\}$ then $x^{*}$ (and hence $\left.y^{*}\right)$ is defined as in (3), while if $x^{*}\left(1_{\mathbf{F}_{2}}, n_{0}, l_{i}\right)=0$ then either $x^{*}\left(1_{\mathbf{F}_{2}}, n_{1}, l_{i}\right)=0$ and we are in case (3) with $\mu=1$ or else $x^{*}\left(1_{\mathbf{F}_{2}}, n_{1}, l_{i}\right)=3$ and we are in case (2). 
REMARK 3.5. The only reason for using the flag 03 before the sequence coding $w \cdot x$ in (2) is to be able to distinguish, assuming $g_{i}$ is bad, whether $\left(x^{*}\left(1_{\mathbf{F}_{2}}, n_{m}, l_{i}\right)\right)_{m}$ is defined as in (2) or (3) only by looking at its first 2 elements. Had we been less stingy with the number of colours and used, say, 5 and 6 instead of 0 and 1 in (2) then we could have avoided using the flag 03 .

We now consider the other case, namely

\section{$g_{i}$ is not a quasi-shift above $l_{i-1}$.}

Let $w_{0}, n_{0}, k_{0}, w_{0}^{\prime}, n_{0}^{\prime}, k_{0}^{\prime}$ be as in cases (i)-(iii). We must define $x^{*}(w, n, k)$ for all $w \in \mathbf{F}_{2}, n \in \mathbb{N}$ and $l_{i-1}<k \leq l_{i}$.

Suppose case (i) holds.

If $k_{0}^{\prime} \leq l_{i-1}$ then set

$$
x^{*}(w, n, k)= \begin{cases}0 & \text { if } x^{*}\left(w_{0}^{\prime}, n_{0}^{\prime}, k_{0}^{\prime}\right)=4, \\ 4 & \text { if } x^{*}\left(w_{0}^{\prime}, n_{0}^{\prime}, k_{0}^{\prime}\right) \leq 3 .\end{cases}
$$

(This is the only way for $x^{*}$ to achieve value 4 .)

If $l_{i-1}<k_{0}^{\prime}$ then set

$$
x^{*}(w, n, k)= \begin{cases}1 & \text { if } k=k_{0}^{\prime} \\ 0 & \text { if } k \neq k_{0}^{\prime}\end{cases}
$$

If case (ii) holds then proceed as in case (i).

If case (iii) holds then set

$$
x^{*}(w, n, k)= \begin{cases}1 & \text { if } n=n_{0}^{\prime}, \\ 0 & \text { if } n \neq n_{0}^{\prime} .\end{cases}
$$

This concludes the definition of $x^{*}$.

We must check that $x \mapsto x^{*}$ is indeed a reduction.

As the value of $x^{*}(w, n, k)$ is independent of $x$ and $w$, when $l_{i-1}<k \leq l_{i}$ and $g_{i}$ is not a quasi-shift, or else depends on $w . x$ only (when $g_{i}$ is a quasishift), it is straightforward to check that

$$
\forall v \in \mathbf{F}_{2} \forall(w, n, k) \in M\left((v \cdot x)^{*}(w, n, k)=x^{*} \circ \widehat{v}(w, n, k)\right) .
$$

We will now show that

$$
\exists i\left(y^{*}=x^{*} \circ g_{i}\right) \Rightarrow \exists v \in \mathbf{F}_{2}(y=v \cdot x)
$$

and hence $x \mapsto x^{*}$ is a reduction. The reader may find useful to refer to Figure 1 while checking the details.

Suppose $y^{*}=x^{*} \circ g_{i}$.

Claim 3.6. $g_{i}$ is a quasi-shift above $l_{i-1}$.

Proof. Deny. Let $w_{0}, n_{0}, k_{0}, w_{0}^{\prime}, n_{0}^{\prime}$, and $k_{0}^{\prime}$ be as in (i)-(iii) above. Notice that their choice as well as the decision as to which of (i)-(iii) we fall into 


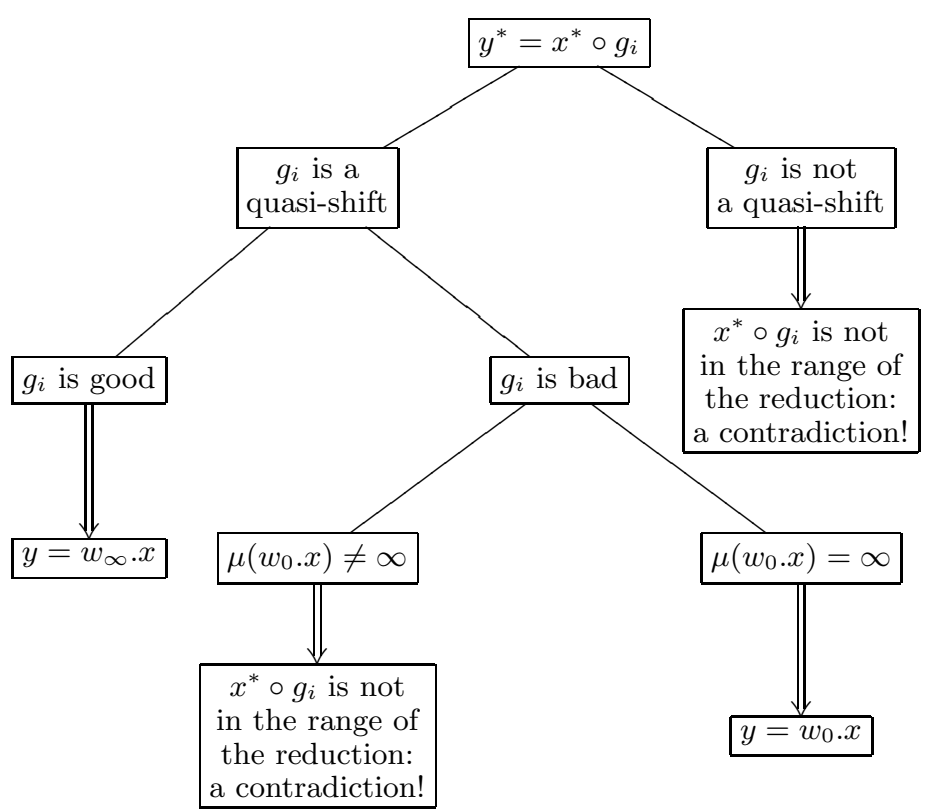

Fig. 1. Plan of the proof of Theorem 3.1

as well as the definition of $x^{*}(w, n, k)$ for $l_{i-1}<k \leq k_{0}$ are independent of $x$. In particular, $x^{*}$ and $y^{*}$ agree on $\left(w_{0}, n_{0}, k_{0}\right)$. If we are in case (i) then

$$
y^{*}\left(w_{0}, n_{0}, k_{0}\right)=x^{*}\left(w_{0}^{\prime}, n_{0}^{\prime}, k_{0}^{\prime}\right) \neq x^{*}\left(w_{0}, n_{0}, k_{0}\right),
$$

and if we are in case (ii) then

$$
x^{*}\left(w_{0}, n_{0}, k_{0}\right)=y^{*}\left(w_{0}^{\prime}, n_{0}^{\prime}, k_{0}^{\prime}\right) \neq y^{*}\left(w_{0}, n_{0}, k_{0}\right),
$$

in both cases reaching a contradiction. If we are in case (iii) then

$$
0=y^{*}\left(w_{0}, n_{0}, l_{i}\right)=x^{*}\left(w_{0}^{\prime}, n_{0}^{\prime}, l_{i}\right)=1,
$$

a contradiction again.

Therefore we may assume that

$g_{i}$ is a quasi-shift.

Again we take cases. Suppose $w_{\infty} \in \mathbf{F}_{2}$ and $\left(n_{m}\right)_{m} \subseteq \mathbb{N}$ witness that $g_{i}$ is good. Then

$$
\begin{aligned}
y^{*}\left(1_{\mathbf{F}_{2}}, n_{m}, l_{i}\right) & =x^{*} \circ g_{i}\left(1_{\mathbf{F}_{2}}, n_{m}, l_{i}\right)=x^{*}\left(w_{\infty}, n_{m}, l_{i}\right) \\
& =\left(w_{\infty} \cdot x\right)^{*}\left(1_{\mathbf{F}_{2}}, n_{m}, l_{i}\right) .
\end{aligned}
$$

As $g_{i}$ is good, both $\left(w_{\infty} \cdot x\right)^{*}$ and $y^{*}$ are defined on $\left\{\left(1_{\mathbf{F}_{2}}, n_{m}, l_{i}\right) \mid m \in \mathbb{N}\right\}$ 
as in (1), hence $\forall m\left(y\left(u_{m}\right)=\left(w_{\infty} \cdot x\right)\left(u_{m}\right)\right)$. Therefore

$$
g_{i} \text { is } \operatorname{good} \Rightarrow y=w_{\infty} \cdot x .
$$

So we may assume that $g_{i}$ is bad and let $\left(w_{m}\right)_{m} \subseteq \mathbf{F}_{2}$ and $\left(n_{m}\right)_{m} \subseteq \mathbb{N}$ witness this so that

$$
\forall m \in \mathbb{N}\left(y^{*}\left(1_{\mathbf{F}_{2}}, n_{m}, l_{i}\right)=x^{*} \circ g_{i}\left(1_{\mathbf{F}_{2}}, n_{m}, l_{i}\right)=x^{*}\left(w_{m}, n_{m}, l_{i}\right)\right) .
$$
hence

Suppose first $\mu\left(w_{0} \cdot x\right)=\infty$. Then by Claim 3.2, $\forall m\left(\left(w_{m} \cdot x\right)^{*}=\left(w_{0} \cdot x\right)^{*}\right)$

$$
x^{*}\left(w_{m}, n_{m}, l_{i}\right)=\left(w_{m} \cdot x\right)^{*}\left(1_{\mathbf{F}_{2}}, n_{m}, l_{i}\right)=\left(w_{0} \cdot x\right)^{*}\left(1_{\mathbf{F}_{2}}, n_{m}, l_{i}\right) .
$$

Since $\left(w_{0} . x\right)^{*}$ is defined on $\left\{\left(1_{\mathbf{F}_{2}}, n_{m}, l_{i}\right) \mid m \in \mathbb{N}\right\}$ as in (2) we see that $y^{*}$ is defined on $\left\{\left(1_{\mathbf{F}_{2}}, n_{m}, l_{i}\right) \mid m \in \mathbb{N}\right\}$ as in (2) and hence $\forall m\left(y\left(u_{m}\right)=\right.$ $\left.w_{0} \cdot x\left(u_{m}\right)\right)$. Therefore

$$
\mu\left(w_{0} \cdot x\right)=\infty \Rightarrow y=w_{0} \cdot x .
$$

Suppose now $1 \leq \mu\left(w_{0} \cdot x\right)=m<\infty$. Then by Claim 3.2,

$\forall j<m\left(\mu\left(w_{j} \cdot x\right)=m \& J_{m}\left(w_{j} \cdot x\right)=J_{m}\left(w_{0} \cdot x\right)\right) \quad$ and $\quad \mu\left(w_{m} \cdot x\right) \leq m$. By (3), the first $m$ elements of the sequence $\left(x^{*} \circ g_{i}\left(1_{\mathbf{F}_{2}}, n_{j}, l_{i}\right)\right)_{j}$ are $(3, \ldots, 3$, $J_{m}\left(w_{0} \cdot x\right)$ ). If $m>1$ (so that the sequence starts with 3 ) or if $J_{m}\left(w_{0} \cdot x\right) \neq 0$ then by Claim 3.4 the next element must be

$$
x^{*}\left(w_{m}, n_{m}, l_{i}\right)=J_{m}\left(w_{0} . x\right) \in\{0,1,2\} .
$$

If $m=1$ and $J_{m}\left(w_{0} \cdot x\right)=0$ then the sequence starts with 0 , hence the next element can only be 0 or 3 , i.e.,

$$
x^{*}\left(w_{m}, n_{m}, l_{i}\right) \in\{0,3\} .
$$

In order to compute this value and eventually reach a contradiction we must consider two cases.

Suppose $\mu\left(w_{m} \cdot x\right)=m$. Since $w_{0} \cdot x=w_{m-1} \cdot x$ and $z_{m} \cdot w_{0} \cdot x=w_{m} \cdot x$ are distinct, they belong to different $X_{m}^{(j)}$ 's and therefore, by (3),

$$
x^{*}\left(w_{m}, n_{m}, l_{i}\right)=J_{m}\left(w_{m} \cdot x\right) \notin\left\{J_{m}\left(w_{0} \cdot x\right), 3\right\},
$$

a contradiction.

Suppose $0<\mu\left(w_{m} \cdot x\right)<m$. Then $m>1$ and by (3) again,

$$
x^{*}\left(w_{m}, n_{m}, l_{i}\right)=3 \neq J_{m}\left(w_{0} \cdot x\right),
$$

a contradiction.

Therefore we have shown that

$$
1 \leq \mu\left(w_{0} \cdot x\right)<\infty \Rightarrow x^{*} \circ g_{i} \text { is not in the range of the reduction. }
$$

This concludes the proof. 
Notice that for $k \geq 5$ or $k=\mathbb{N}$ and $G$ as in the theorem, the inclusion map $5^{\mathbb{N}} \hookrightarrow k^{\mathbb{N}}$ witnesses $\cong_{G}^{k}$ is universal. Focusing on Rec, the group of recursive permutations, one can show that

$$
\cong \text { Rec }\left\lceil\left\{x \in \mathbb{N}^{\mathbb{N}} \mid x: \mathbb{N} \rightarrow \mathbb{N} \text { is onto }\right\}\right.
$$

is universal. To see this assign to each non-empty subset $S \subseteq 5=\{0, \ldots, 4\}$ an integer $\varrho(S)>0$, and consider the map $5^{\mathbb{N}} \rightarrow\left\{x \in \mathbb{N}^{\mathbb{N}} \mid x: \mathbb{N} \rightarrow \mathbb{N}\right.$ is onto $\}, x \mapsto x^{*}$, defined as follows: on the even integers we copy $x$, i.e. $\forall n\left(x^{*}(2 n)=x(n)\right)$, and on the odd integers we start with $\varrho($ range $(x))$ consecutive 5's followed by the increasing enumeration of $\mathbb{N} \backslash($ range $(x) \cup\{5\})$. It is easy to check that $x^{*}$ is indeed onto and that $x \mapsto x^{*}$ is Borel. If $x \circ f=y$ for some recursive $f$ then letting $g(2 n+1)=2 n+1$ and $g(2 n)=2 f(n)$ we find that $x^{*} \circ g=y^{*}$. Suppose instead $x^{*} \circ f=y^{*}$ for some recursive bijection $f$. Since the numbers of 5's in $x^{*}$ and in $x^{*} \circ f=y^{*}$ are the same, range $(x)=\operatorname{range}(y)$ and hence $f$ restricted to the even numbers must be a bijection. Letting $h(n)=\frac{1}{2} f(2 n)$ we see that $x \circ h=y$. Therefore $x \mapsto x^{*}$ is a reduction of $\cong_{\text {Rec }}^{5}$ to $\cong_{\operatorname{Rec}}^{\mathbb{N}}\left\lceil\left\{x \in \mathbb{N}^{\mathbb{N}} \mid x: \mathbb{N} \rightarrow \mathbb{N}\right.\right.$ is onto $\}$.

By extracting the salient features of Rec used above we get

Corollary 3.7. Let $G_{0} \subseteq G \subseteq S_{\infty}$ be as in the theorem. Suppose $G$ has the following closure property:

- if $f \in G$ then $g \in G$ where $g(2 n+1)=2 n+1$ and $g(2 n)=2 f(n)$,

- if $f \in G$ and $f$ is a bijection when restricted to the set of even numbers (i.e., $\forall n \exists m(f(2 m)=2 n)$ and $\forall n \exists m(f(2 n)=2 m))$ then $h \in G$ where $h(n)=\frac{1}{2} f(2 n)$.

Then $\cong_{G}^{\mathbb{N}}\left\lceil\left\{x \in \mathbb{N}^{\mathbb{N}} \mid x: \mathbb{N} \rightarrow \mathbb{N}\right.\right.$ is onto $\}$ is universal.

Let $\mathcal{L}$ be a language consisting of one binary relation symbol and let $X_{\mathcal{L}}=2^{\mathbb{N}^{2}}$ be the space of all $\mathcal{L}$-structures with universe $\mathbb{N}$. Let $\operatorname{Eq}(k) \subseteq X_{\mathcal{L}}$ be the set of all structures which are models for equivalence relations whose equivalence classes are of size at most $k$, that is, $x \in \mathrm{Eq}(k)$ iff the relation $E^{x}$ on $\mathbb{N}$ defined by

$$
n E^{x} m \Leftrightarrow x(n, m)=1
$$

is an equivalence relation on $\mathbb{N}$ and $\forall n \in \mathbb{N}\left(\left|[n]_{E^{x}}\right| \leq k\right)$. For $x, y \in X_{\mathcal{L}}$ set $x \cong_{G}^{\mathcal{L}} y$ just in case the models $\left\langle\mathbb{N}, E^{x}\right\rangle$ and $\left\langle\mathbb{N}, E^{y}\right\rangle$ are isomorphic via a bijection in $G$.

For $G \subseteq S_{\infty}$ let $\sim_{G}$ denote the conjugation relation on $S_{\infty}$ via elements in $G$

$$
x \sim_{G} y \Leftrightarrow \exists g \in G\left(g^{-1} \circ x \circ g=y\right) .
$$

Let also $\sim_{G}^{k}=\sim_{G} \uparrow\left\{x \in S_{\infty} \mid x\right.$ is a product of cycles of length $\left.\leq k\right\}$. 
A group $G \subseteq S_{\infty}$ is closed under pairing if there is a pairing function, i.e., a bijection $j: \mathbb{N}^{2} \rightarrow \mathbb{N}$ with inverses $(j(n, m))_{0}=n$ and $(j(n, m))_{1}=m$ such that:

For every $g \in G$ the bijection $h \in S_{\infty}$ defined by

$$
h(j(n, m))=j(g(n), m)
$$

is in $G$, and if

$$
f(n)=(g(j(n, 0)))_{0}
$$

is a bijection, then $f$ is also in $G$. For example, Rec is closed under pairing.

Proposition 3.8. Let $G \subseteq S_{\infty}$ be a countable group closed under pairing. Then

$$
\cong_{G}^{k} \sqsubseteq_{\mathrm{c}} \cong_{G}^{\mathcal{L}} \uparrow \mathrm{Eq}(k+1),
$$

that is to say: the equivalence relation given by the right action of $G$ on $k^{\mathbb{N}}$ is continuously embeddable into the $G$-isomorphism relation between equivalence relations on $\mathbb{N}$ with equivalence classes of size $\leq k+1$.

Similarly, $\cong_{G}^{k}$ continuously embeds into conjugacy on $S_{\infty}$ via elements of $G$ restricted to products of cycles of size $\leq k+1$ :

$$
\cong_{G}^{k} \sqsubseteq_{\mathrm{c}} \sim_{G}^{k+1} \text {. }
$$

In particular, for all $G_{0} \subseteq G \subseteq S_{\infty}$ as in the theorem, $\cong_{G}^{\mathcal{L}} \uparrow \mathrm{Eq}(6)$ and $\sim_{G}^{6}$ are both universal.

Proof. Let $\vartheta: k^{\mathbb{N}} \rightarrow \mathrm{Eq}(k+1)$ be defined by

$$
j(n, m) E^{\vartheta(x)} j\left(n^{\prime}, m^{\prime}\right) \Leftrightarrow n=n^{\prime} \&\left(m=m^{\prime} \vee m, m^{\prime} \leq x(n)+1\right)
$$

where $j$ is the pairing function for $G$. (It is most convenient to visualize $E^{\vartheta(x)}$ as the equivalence relation on $\mathbb{N}^{2}$ obtained by connecting on each vertical line $\{(n, m) \mid m \in \mathbb{N}\}$ all the points $\leq x(n)+1$.) It is immediate to check that $\vartheta(x) \in \mathrm{Eq}(k+1)$ and that $\vartheta$ is continuous and injective.

Suppose first $x, y \in k^{\mathbb{N}}$ and $x \circ g=y$ for some $g \in G$. Let $h \in G$ be defined by $h(j(n, m))=j(g(n), m)$. Then

$$
\begin{aligned}
h(j(n, m)) E^{\vartheta(x)} h\left(j\left(n^{\prime}, m^{\prime}\right)\right) & \Leftrightarrow j(g(n), m) E^{\vartheta(x)} j\left(g\left(n^{\prime}\right), m^{\prime}\right) \\
& \Leftrightarrow j(n, m) E^{\vartheta(y)} j\left(n^{\prime}, m^{\prime}\right),
\end{aligned}
$$

that is, $\vartheta(y) \cong{ }_{G}^{\mathcal{L}} \vartheta(x)$ via $h$.

Conversely, suppose $\vartheta(y) \cong{ }_{G}^{\mathcal{L}} \vartheta(x)$ via some $g \in G$. Let $f \in G$ be defined by $f(n)=(g(j(n, 0)))_{0}$. We must show that $x \circ f=y$. Fix $n \in \mathbb{N}$. Since $[j(n, 0)]_{E \vartheta(y)}$ has $y(n)+1$ elements and $g$ is an isomorphism between $E^{\vartheta(y)}$ and $E^{\vartheta(x)}$, we see that $[g(j(n, 0))]_{E^{\vartheta(x)}}$ also has $y(n)+1$ elements. In particular, $[g(j(n, 0))]_{E^{\vartheta(x)}}$ is not a singleton. But $g(j(n, 0))=j(f(n), l)$ for some $l$, so $[j(f(n), l)]_{E^{\vartheta(x)}}=[j(f(n), 0)]_{E^{\vartheta(x)}}$ has $x(f(n))+1$ elements. Therefore $y(n)=x(f(n))$, which is what we had to prove. 
For $\sim_{G}^{k+1}$ we use essentially the same reduction: for $x \in k^{\mathbb{N}}$ let $\varphi(x) \in S_{\infty}$ be defined by $\varphi(x)=\prod_{n} c_{n}(x)$ where $c_{n}(x)$ is the cycle

$$
j(n, 0) \longmapsto j(n, 1) \longmapsto \cdots \longmapsto j(n, x(n)+1) .
$$

It is immediate to check that $x \cong{ }_{G}^{k} y \Leftrightarrow \varphi(x) \sim_{G}^{k+1} \varphi(y)$.

Even if the theorem holds for $k=2$ (as we conjecture) the proposition above does not yield any information about the universality of $\cong \mathcal{L}_{G}^{\mathcal{L}} \uparrow \mathrm{Eq}(2)$. We can still though relate this equivalence relation to $\sim_{G}$.

For $x \in \mathrm{Eq}(2)$ let $x^{*} \in S_{\infty}$ be defined by

$$
x^{*}(n)= \begin{cases}m & \text { if }[n]_{E^{x}}=\{n, m\} \text { and } n \neq m, \\ n & \text { if }[n]_{E^{x}}=\{n\} .\end{cases}
$$

Then $x^{*} \in I:=\left\{f \in S_{\infty} \mid f \circ f=\mathrm{id}\right\}$, the set of all involutions, and the map $\operatorname{Eq}(2) \rightarrow I, x \mapsto x^{*}$, is clearly a Borel bijection. Moreover, if $G \subseteq S_{\infty}$ is a subgroup, $g \in G$, and $x, y \in \operatorname{Eq}(2)$ then $g:\left\langle\mathbb{N}, E^{x}\right\rangle \rightarrow\left\langle\mathbb{N}, E^{y}\right\rangle$ is an isomorphism iff $g \circ x^{*} \circ g^{-1}=y^{*}$. Therefore we have proved

Proposition 3.9. Let $G \subseteq S_{\infty}$ be a subgroup. The logic action of $G$ on $\mathrm{Eq}(2)$ is isomorphic to the conjugacy of $G$ on elements of $I$. In particular,

$$
\simeq_{G}^{\mathcal{L}}\left\lceil\mathrm{Eq}(2) \cong_{\mathrm{B}} \sim_{G}\lceil I\right.
$$

4. Conjugacy of subgroups. We will now prove Theorem 1.3. The proof will use a combination of the techniques used in proving 3.1 together with a few facts about free groups.

Let $\mathbf{F}(X)$ be the free group on the set $X \neq \emptyset$, let $x \in X$ and let $w \in$ $\mathbf{F}(X)$. Let $x_{1}^{k_{1}} \ldots x_{n}^{k_{n}}$ be the unique reduced word for $w$. Then we say that $w$ starts with $x_{1}$ and ends with $x_{n}$. Whenever we write " $v w$ is a reduced word" we mean that both $v$ and $w$ are already written as reduced words, and for no $x \in X, v$ ends with $x$ and $w$ starts with $x$. We say that $x^{k}$ occurs in $w(k \in \mathbb{Z} \backslash\{0\})$ if $w=v_{0} x^{k} v_{1}$ and $v_{0} x^{k} v_{1}$ is a reduced word, i.e., $v_{0}$ and $v_{1}$ are reduced and neither $v_{0}$ ends with $x$ nor $v_{1}$ begins with $x$. If $x^{k}$ occurs in $w$ then we say that $x$ is a generator in $w$. It is easy to see that if $x$ is a generator in $w$, then it is a generator in every $w^{n}$, for $n \neq 0$. A word $w$ is symmetric if it can be written in reduced form as $w=v_{0} x^{k} v_{0}^{-1}$; otherwise it is asymmetric.

LEMMA 4.1. If $w \in \mathbf{F}(X)$ is asymmetric then there is a fixed bound $M$ depending on $w$ such that if $x^{k}$ occurs in any $w^{n}$ then $|k| \leq M$, i.e.,

$$
\exists M>0 \forall x \in X \forall k \in \mathbb{Z} \backslash\{0\} \forall n\left(x^{k} \text { occurs in } w^{n} \Rightarrow|k| \leq M\right) \text {. }
$$

Proof. It is enough to show that for any $x \in X$ which is a generator of $w$ there is an $M_{x}$ such that if $x^{h}$ occurs in $w^{n}$, then $|h| \leq M_{x}$, so that we 
can set

$$
M=\sup \left\{M_{x} \mid x \text { is a generator in } w\right\} .
$$

Let $x$ be a generator in $w$ so that $w=v_{0} x^{k} v_{1}$ is a reduced word and $v_{1} \neq v_{0}^{-1}$. Then the reduced form of $v_{1} v_{0}$ cannot start and end with $x$ since otherwise either $v_{1}$ would have to start with $x$ or $v_{0}$ would have to end with $x$, hence $v_{0} x^{k} v_{1}$ would not be reduced. Consider the product

$$
w^{n}=v_{0} x^{k} v_{1} \cdot v_{0} x^{k} v_{1} \ldots v_{0} x^{k} v_{1} .
$$

After the reduction:

- if $v_{1} \cdot v_{0}$ in reduced form does not start or end with $x$, then the boxed occurrences of $x^{k}$ remain unchanged, so let

$$
M_{x}=\max \left(|k|, \sup \left\{|h| \mid x^{h} \text { occurs in } v_{0} \text {, or in } v_{1} \text {, or in } v_{1} v_{0}\right\}\right) \text {, }
$$

- if $v_{1} \cdot v_{0}$ in reduced form is $u x^{h}$ or $x^{h} u$, then the first (respectively: the last) boxed occurrence of $x^{k}$ remains unchanged, so let

$$
M_{x}=\max \left(|k|,|k+h|, \sup \left\{|j| \mid x^{j} \text { occurs in } v_{0} \text {, or in } v_{1} \text {, or in } u\right\}\right) \text {. }
$$

Proof of Theorem 1.3. Since inside every free group on two generators there is a copy of the free group on three generators, pick independent elements $a, b, c \in G$ so that $\langle a, b, c\rangle$ is a free group on 3 generators. Let $\mathbf{F}_{3}=\langle a, b, c\rangle$ be this particular realization of the free group on 3 generators and let $\mathbf{F}_{2}=\langle a, b\rangle \subset \mathbf{F}_{3}$. We want to construct a copy of $\mathbf{F}_{\omega}$, the free group of rank $\aleph_{0}$, inside $\mathbf{F}_{3}$. We need the following lemma.

LemMa 4.2. Let $w_{1}, \ldots, w_{n} \in \mathbf{F}_{2}$ be distinct. Then $\left\langle w_{1} c w_{1}^{-1}, \ldots\right.$, $\left.w_{n} c w_{n}^{-1}\right\rangle$ is a free group on $n$ generators. In particular, if $\left(w_{n}\right)_{n} \subseteq \mathbf{F}_{2}$ are all distinct then $\left\langle w_{1} c w_{1}^{-1}, w_{2} c w_{2}^{-1}, \ldots\right\rangle$ is a free group of rank $\aleph_{0}$.

Proof. Choose new letters $\sigma_{1}, \ldots, \sigma_{n}$, and let $\left\langle\sigma_{1}, \ldots, \sigma_{n}\right\rangle$ be the free group of rank $n$ generated by them. It is enough to $\operatorname{show}$ that $\operatorname{ker}(\varphi)$ is null, where $\varphi:\left\langle\sigma_{1}, \ldots, \sigma_{n}\right\rangle \rightarrow\left\langle w_{1} c w_{1}^{-1}, \ldots, w_{n} c w_{n}^{-1}\right\rangle$ is the epimorphism induced by the map $\sigma_{i} \mapsto w_{i} c w_{i}^{-1}$. Let $v=\sigma_{i_{1}}^{k_{1}} \sigma_{i_{2}}^{k_{2}} \ldots \sigma_{i_{m}}^{k_{m}}$ be a reduced word of $\left\langle\sigma_{1}, \ldots, \sigma_{n}\right\rangle$. If

$$
\varphi(v)=w_{i_{1}} c^{k_{1}} w_{i_{1}}^{-1} \cdot w_{i_{2}} c^{k_{2}} w_{i_{2}}^{-1} \ldots w_{i_{m}} c^{k_{m}} w_{i_{m}}^{-1}=1_{\mathbf{F}_{2}}
$$

and $m>1$, then $w_{i_{j}}=w_{i_{j+1}}$ for all $1 \leq j<m$, hence $\sigma_{i_{j}}=\sigma_{i_{j+1}}$. Therefore $m=1, v=\sigma_{i_{1}}^{k_{1}}$ and hence $\varphi(v)=w_{i_{1}} c^{k_{1}} w_{i_{1}}^{-1}=1_{\mathbf{F}_{2}}$ implies $k_{1}=0$. This contradicts the assumption that $v$ is reduced.

Define also the free group on two generators $\widetilde{\mathbf{F}}_{2}=\left\langle a^{2}, b^{2}\right\rangle \subset \mathbf{F}_{2}$, and let $w \mapsto \widetilde{w}$ be the isomorphism $\mathbf{F}_{2} \rightarrow \widetilde{\mathbf{F}}_{2}$ given by $a \mapsto a^{2}$ and $b \mapsto b^{2}$. For any $w \in \mathbf{F}_{2}$ and $n \in \mathbb{Z}$ let

$$
\gamma(w, n)=\widetilde{w}^{-1} b a^{2 n} c a^{-2 n} b^{-1} \widetilde{w} \in \mathbf{F}_{3} .
$$


Let $\Gamma=\bigcup_{w \in \mathbf{F}_{2}} \Gamma_{w} \subset \mathbf{F}_{3}$, where $\Gamma_{w}=\{\gamma(w, n) \mid n \in \mathbb{Z}\}$. Suppose $\gamma(w, n)=$ $\gamma(z, m)$. Then

$$
\widetilde{w}^{-1} b a^{2 n} c a^{-2 n} b^{-1} \widetilde{w}=\widetilde{z}^{-1} b a^{2 m} c a^{-2 m} b^{-1} \widetilde{z} .
$$

As there is only one occurrence of $c$ on both sides of the equation, we have

$$
\widetilde{w}^{-1} b a^{2 n}=\widetilde{z}^{-1} b a^{2 m} .
$$

As $\widetilde{w}, \widetilde{z} \in \widetilde{\mathbf{F}}_{2}$, it is easy to see that $w=z$ and $n=m$. Therefore

$$
\forall w, z \in \mathbf{F}_{2}\left(w \neq z \Rightarrow \Gamma_{z} \cap \Gamma_{w}=\emptyset\right) .
$$

Since $\gamma(w, n)=\left(\widetilde{w}^{-1} b a^{2 n}\right) c\left(\widetilde{w}^{-1} b a^{2 n}\right)^{-1}$ and $\widetilde{w}^{-1} b a^{2 n} \in \mathbf{F}_{2}$, Lemma 4.2 implies that $\Gamma$ is a countable set of independent elements and

$$
\mathbf{F}_{\omega}=\langle\Gamma\rangle \subset \mathbf{F}_{3} \subseteq G
$$

is a free group on $\aleph_{0}$ generators. We now concentrate on subgroups of $\mathbf{F}_{\omega}$ and from now on words will mean words built from elements in $\Gamma$. Notice that in our set-up $\mathbf{F}_{2} \nsubseteq \mathbf{F}_{\omega}$.

The plan is to associate, in a Borel way, with each $x \in 2^{\mathbf{F}_{2}}$ a group $H(x) \subseteq \mathbf{F}_{\omega}$ such that $H(x) \cong \mathbf{F}_{\omega}$ and

$$
\begin{gathered}
\forall w \in \mathbf{F}_{2} \forall x \in 2^{\mathbf{F}_{2}}\left(H(w \cdot x)=\widetilde{w} H(x) \widetilde{w}^{-1}\right), \\
\forall g \in G \forall x, y \in 2^{\mathbf{F}_{2}}\left(H(y)=g H(x) g^{-1} \Rightarrow \exists w \in \mathbf{F}_{2}(w \cdot x=y)\right) .
\end{gathered}
$$

This implies that the shift action of $\mathbf{F}_{2}$ on $2^{\mathbf{F}_{2}}$ is reducible to $E_{\mathrm{c}}(G, \operatorname{Sgr}(G))$, proving the universality of the latter.

The group $H(x)$ will be given via an infinite set $\mathcal{G}(x)$ of generators so that

$$
\begin{aligned}
& \mathcal{G}(x) \text { is an independent set of elements of } \mathbf{F}_{\omega} \text { and }\langle\mathcal{G}(x)\rangle=H(x) \\
& \cong \mathbf{F}_{\omega} \text {. }
\end{aligned}
$$

In order to define the $\mathcal{G}(x)$ 's we need to re-arrange the enumeration of $\Gamma$ a bit: fix a bijection $j: \mathbb{N}^{2} \rightarrow \mathbb{Z}$ and set

$$
\alpha(w, n, m)=\gamma(w, j(n, m)) .
$$

The third component of the indexing gives the level of the elements of $\Gamma$, so we say that $\alpha(w, n, m)$ is of level $m$. Fix for every $i>0$ an infinite set $S_{i} \subseteq \mathbb{P}$ (= the set of all primes) such that $i \neq j \Rightarrow S_{i} \cap S_{j}=\emptyset$. As in the proof of 3.1 an increasing sequence of integers $\left(l_{i}\right)_{i \in \mathbb{N}}$ with $l_{0}=-1$ will be defined inductively. At stage $i$ the only elements added to $\mathcal{G}(x)$ will be of the form $\gamma^{p}$, where $\gamma \in \Gamma$ is of level between $l_{i-1}$ and $l_{i}$ and $p$ is some unique element of $S_{i}$; moreover, some such element should be added at this stage:

$$
\begin{aligned}
& \forall z \in \mathcal{G}(x) \exists \alpha(w, n, m) \in \Gamma \exists p\left(\alpha(w, n, m)^{p}=z \&\left(l_{i-1}<m \leq l_{i} \Rightarrow\right.\right. \\
& \left.\left.p \in S_{i}\right)\right) \& \forall \gamma \in \Gamma \forall p, q \in \mathbb{P}\left(\gamma^{p}, \gamma^{q} \in \mathcal{G}(x) \Rightarrow p=q\right) . \\
& \exists \alpha(w, n, m) \in \Gamma \exists p \in S_{i}\left(l_{i-1}<m \leq l_{i} \& \alpha(w, n, m)^{p} \in \mathcal{G}(x)\right) .
\end{aligned}
$$


(4) $\forall w \in \mathbf{F}_{2}\left(\widetilde{w} \mathcal{G}(x) \widetilde{w}^{-1}=\mathcal{G}(w \cdot x)\right)$.

(5) If $w \in H(x)$ and $\alpha(w, n, m)^{k}$ occurs in $w$ and $l_{i-1}<m \leq l_{i}$ then

$$
\exists p \in S_{i}\left(p \mid k \& \alpha(w, n, m)^{p} \in \mathcal{G}(x)\right) .
$$

In particular, $\Gamma \cap \mathcal{G}(x)=\emptyset$. By (2) the prime $p$ is unique.

(6) If $v w$ is a reduced word then $v w \in H(x) \Rightarrow v, w \in H(x)$.

Notice that properties (2) and (3) imply properties (1) and (5), property (6) follows from property (5), and as $\widetilde{z}^{-1} \gamma(w, n)^{k} \widetilde{z}=\gamma(w z, n)^{k}$ for any $z, w \in \mathbf{F}_{2}$ and $n, k \in \mathbb{Z}$, property (4) will hold.

We now start the construction.

In analogy with the proof of 3.1 , we say that an element $g \in G$ is a quasi-shift above $l$ if

$$
\begin{aligned}
& \forall w, n\left(g^{-1} \cdot \alpha(w, n, l+1) \cdot g \in \mathbf{F}_{\omega}\right. \\
& \& \text { every generator from } \Gamma \text { in } g^{-1} \cdot \alpha(w, n, l+1) \cdot g \\
& \text { is either of the form } \alpha(z, m, k) \text { with } k \leq l \\
& \text { or else of the form } \alpha(z, n, l+1) \\
& \left.\& \exists z \alpha(z, n, l+1) \text { is a generator in } g^{-1} \cdot \alpha(w, n, l+1) \cdot g\right) .
\end{aligned}
$$

Fix an enumeration $\left(g_{i}\right)_{i \geq 1}$ of $G$.

Set $l_{0}=-1$ and suppose $l_{i-1}$ has been defined for some $i \geq 1$. We now distinguish two cases:

- $g_{i}$ is not a quasi-shift above $l_{i-1}$. Then letting $k_{0}=l_{i-1}+1$, we have the following possibilities:

(i) $\exists w_{0}, n_{0}\left(g_{i}^{-1} \cdot \alpha\left(w_{0}, n_{0}, k_{0}\right) \cdot g_{i} \notin \mathbf{F}_{\omega}\right)$.

(ii) Case (i) fails and

$$
\begin{aligned}
& \exists w_{0}, n_{0}, w_{0}^{\prime}, n_{0}^{\prime}, k_{0}^{\prime}\left(k_{0}^{\prime}>k_{0}\right. \\
& \left.\& \alpha\left(w_{0}^{\prime}, n_{0}^{\prime}, k_{0}^{\prime}\right) \text { is a generator in } g_{i}^{-1} \cdot \alpha\left(w_{0}, n_{0}, k_{0}\right) \cdot g_{i}\right) .
\end{aligned}
$$

(iii) Cases (i) and (ii) fail and

$$
\begin{aligned}
& \exists w_{0}, n_{0}, w_{0}^{\prime}, n_{0}^{\prime}\left(n_{0} \neq n_{0}^{\prime}\right. \\
& \left.\& \alpha\left(w_{0}^{\prime}, n_{0}^{\prime}, k_{0}\right) \text { is a generator in } g_{i}^{-1} \cdot \alpha\left(w_{0}, n_{0}, k_{0}\right) \cdot g_{i}\right) .
\end{aligned}
$$

(iv) Cases (i)-(iii) fail and

$$
\begin{array}{r}
\exists w_{0}, n_{0} \forall w_{0}^{\prime}, n_{0}^{\prime}, k_{0}^{\prime}\left(\alpha\left(w_{0}^{\prime}, n_{0}^{\prime}, k_{0}^{\prime}\right) \text { is a generator in } g_{i}^{-1} \cdot \alpha\left(w_{0}, n_{0}, k_{0}\right) \cdot g_{i}\right. \\
\left.\Rightarrow k_{0}^{\prime}<k_{0}\right) .
\end{array}
$$

Then set $l_{i}=\max \left(k_{0}, k_{0}^{\prime}\right)$. (Notice that if $g_{i}$ is not a quasi-shift above $l_{i-1}$ because of (iv) then all of the generators of $g_{i}^{-1} \cdot \alpha\left(w_{0}, n_{0}, k_{0}\right) \cdot g_{i}$ are of level $<k_{0}$.) 
- Otherwise, that is: $g_{i}$ is a quasi-shift above $l_{i-1}$. In this case set $l_{i}=$ $l_{i-1}+1$.

Suppose $g_{i}$ is a quasi-shift above $l_{i-1}$. We say that $g_{i}$ is good if there is an increasing sequence $\left(n_{m}\right)_{m}$ of natural numbers and a $w_{\infty} \in \mathbf{F}_{2}$ such that

$$
\alpha\left(w_{\infty}, n_{m}, l_{i}\right) \text { is a generator in } g_{i}^{-1} \cdot \alpha\left(1_{\mathbf{F}_{2}}, n_{m}, l_{i}\right) \cdot g_{i} .
$$

If $g_{i}$ is not good then it is called bad. In this case there is an increasing sequence $\left(n_{m}\right)_{m}$ of natural numbers and a sequence $\left(w_{m}\right)_{m}$ of distinct elements in $\mathbf{F}_{2}$ such that

$$
\forall m \in \mathbb{N}\left(\alpha\left(w_{m}, n_{m}, l_{i}\right) \text { is a generator in } g_{i}^{-1} \cdot \alpha\left(1_{\mathbf{F}_{2}}, n_{m}, l_{i}\right) \cdot g_{i}\right) .
$$

Let also $\left(z_{m}\right)_{m \geq 1}, \mu$ and $J_{m}$ be defined as in the proof of 3.1 .

Finally, we define the map $x \mapsto \mathcal{G}(x)$, and hence the reduction $H: 2^{\mathbf{F}_{2}} \rightarrow$ $\operatorname{Sgr}(G), x \mapsto H(x)$, so that properties (1)-(6) hold.

\section{$g_{i}$ is not a quasi-shift above $l_{i-1}$.}

Let $w_{0}, n_{0}, k_{0}, w_{0}^{\prime}, n_{0}^{\prime}, k_{0}^{\prime}$ be as in cases (i)-(iv).

Suppose case (i) holds. Then $l_{i}=k_{0}$. Let $r$ be the least positive integer such that $g_{i}^{-1} \cdot \alpha\left(w_{0}, n_{0}, k_{0}\right)^{r} \cdot g_{i} \in \mathbf{F}_{\omega}$ if such an integer exists, or $r=0$ otherwise. Let $p=\min \left(S_{i} \backslash\{r\}\right)$ be the least element of $S_{i}$ different from $r$. We now require in our construction that

$$
\forall x \in 2^{\mathbf{F}_{2}} \forall w \in \mathbf{F}_{2}\left(\alpha\left(w, n_{0}, l_{i}\right)^{p} \in \mathcal{G}(x)\right) .
$$

Suppose case (ii) holds. Then $k_{0}=l_{i-1}+1<l_{i}$. Let $p=\min \left(S_{i}\right)$ and set

$$
\forall x \in 2^{\mathbf{F}_{2}} \forall w \in \mathbf{F}_{2}\left(\alpha\left(w, n_{0}, k_{0}\right)^{p} \in \mathcal{G}(x)\right) .
$$

Suppose case (iii) or (iv) holds. Let $p=\min \left(S_{i}\right)$ and set

$$
\forall x \in 2^{\mathbf{F}_{2}} \forall w \in \mathbf{F}_{2}\left(\alpha\left(w, n_{0}, l_{i}\right)^{p} \in \mathcal{G}(x)\right) .
$$

For $k \in\left(l_{i-1}, l_{i}\right]$ and $n \in \mathbb{N}, p \in \mathbb{P}$ place $\alpha(w, n, k)^{p}$ in $\mathcal{G}(x)$ only if required by the cases above.

We now consider the other case, namely

$$
g_{i} \text { is a quasi-shift above } l_{i-1} \text {. }
$$

There are two possibilities.

- For some $\alpha\left(w_{0}, n_{0}, l_{i}\right) \in \Gamma$, the reduced word $v$ obtained from $g_{i}^{-1}$. $\alpha\left(w_{0}, n_{0}, l_{i}\right) \cdot g_{i}$ is either asymmetric or else it is symmetric of the form $v_{1} \cdot \alpha\left(w_{0}^{\prime}, n_{0}^{\prime}, k\right)^{m} \cdot v_{1}^{-1}$ with $k<l_{i}$ and $m \neq 0$. In either case, by Lemma 4.1 or by inspection, there is $M$ such that

$$
\forall r\left(\alpha\left(z, n_{0}, l_{i}\right)^{k} \text { occurs in } v^{r} \Rightarrow|k| \leq M\right) .
$$


When this happens $g_{i}$ is said to be bounded. Let $p$ be the least element in $S_{i}$ larger than $M$ and set

$$
\forall x \in 2^{\mathbf{F}_{2}} \forall w \in \mathbf{F}_{2}\left(\alpha\left(w, n_{0}, l_{i}\right)^{p} \in \mathcal{G}(x)\right) .
$$

- Otherwise $\forall w \in \mathbf{F}_{2} \forall n \in \mathbb{N} \exists z \in \mathbf{F}_{2} \exists u \in \mathbf{F}_{\omega}$ such that

$$
g_{i}^{-1} \cdot \alpha\left(w, n, l_{i}\right) \cdot g_{i}=u \cdot \alpha\left(z, n, l_{i}\right)^{r(w, n)} \cdot u^{-1}
$$

where $r(w, n) \in \mathbb{Z} \backslash\{0\}$. In this case $g_{i}$ is said to be unbounded.

CASE 1: $\exists w_{0}, n_{0}\left(\left|r\left(w_{0}, n_{0}\right)\right|>1\right)$, that is, for some $w_{0}, n_{0}, z, u$,

$$
g_{i}^{-1} \cdot \alpha\left(w_{0}, n_{0}, l_{i}\right) \cdot g_{i}=u \cdot \alpha\left(z, n_{0}, l_{i}\right)^{r} \cdot u^{-1}
$$

with $r=r\left(w_{0}, n_{0}\right)$ and $|r|>1$. Then let $p=\min \left(S_{i}\right)$ and set

$$
\forall x \in 2^{\mathbf{F}_{2}} \forall w \in \mathbf{F}_{2}\left(\alpha\left(w, n_{0}, l_{i}\right)^{p} \in \mathcal{G}(x)\right) .
$$

CASE 2: $\forall w \in \mathbf{F}_{2} \forall n \in \mathbb{N}(|r(w, n)|=1)$, that is,

$\forall w \in \mathbf{F}_{2} \forall n \in \mathbb{N} \exists z \in \mathbf{F}_{2} \exists u \in \mathbf{F}_{\omega}\left(g_{i}^{-1} \cdot \alpha\left(w, n, l_{i}\right) \cdot g_{i}=u \cdot \alpha\left(z, n, l_{i}\right)^{ \pm 1} \cdot u^{-1}\right)$.

Then we can mimic the construction of 3.1. Fix an enumeration $\left(u_{m}\right)_{m}$ of $\mathbf{F}_{2}$ without repetitions. Let $p_{0}, \ldots, p_{5}$ be the first six elements of $S_{i}$. We distinguish three possibilities.

- $g_{i}$ is good. Then $\exists w_{\infty} \exists\left(n_{m}\right)_{m} \exists\left(v_{m}\right)_{m}$ such that $\left(n_{m}\right)_{m}$ is increasing and $g_{i}^{-1} \cdot \alpha\left(1_{\mathbf{F}_{2}}, n_{m}, l_{i}\right) \cdot g_{i}=v_{m} \cdot \alpha\left(w_{\infty}, n_{m}, l_{i}\right)^{ \pm 1} \cdot v_{m}^{-1}$. By thinning down the sequence we may assume that the exponent is constant, say 1 . Set

$$
\begin{aligned}
\alpha\left(w, n_{m}, l_{i}\right)^{p_{1}} & \in \mathcal{G}(x) \Leftrightarrow w \cdot x\left(u_{m}\right)=1, \\
\alpha\left(w, n, l_{i}\right)^{p_{0}} & \in \mathcal{G}(x) \Leftrightarrow\left(n=n_{m} \& w \cdot x\left(u_{m}\right)=0\right) \vee n \notin\left\{n_{m} \mid m \in \mathbb{N}\right\} .
\end{aligned}
$$

So we may assume that $g_{i}$ is bad hence $\exists\left(w_{m}\right)_{m} \exists\left(n_{m}\right)_{m} \exists\left(v_{m}\right)_{m}$ such that the $n_{m}$ 's are increasing, the $w_{m}$ 's are distinct, and $g_{i}^{-1} \cdot \alpha\left(1_{\mathbf{F}_{2}}, n_{m}, l_{i}\right)$. $g_{i}=v_{m} \cdot \alpha\left(w_{m}, n_{m}, l_{i}\right)^{ \pm 1} \cdot v_{m}^{-1}$. Again by thinning down we may assume that the exponent is 1 .

- $g_{i}$ is bad and $\mu(w \cdot x)=\infty$. Set

$$
\begin{aligned}
\alpha\left(w, n_{m}, l_{i}\right)^{p_{1}} & \in \mathcal{G}(x) \Leftrightarrow w \cdot x\left(u_{n}\right)=1, \\
\alpha\left(w, n, l_{i}\right)^{p_{0}} & \in \mathcal{G}(x) \Leftrightarrow\left(n=n_{m} \& w \cdot x\left(u_{n}\right)=0\right) \vee n \notin\left\{n_{m} \mid m \in \mathbb{N}\right\} .
\end{aligned}
$$

- $g_{i}$ is bad and $\mu(w . x)<\infty$. Then set

$$
\alpha\left(w, n, l_{i}\right)^{p_{j}} \in \mathcal{G}(x),
$$

where

$$
j= \begin{cases}0 & \text { if } n \notin\left\{n_{m} \mid m \in \mathbb{N}\right\} \\ 2 & \text { if } n=n_{m} \text { and } m<\mu(w \cdot x)-1 \text { or } m>\mu(w . x), \\ J_{\mu(w . x)}(w \cdot x)+3 & \text { if } n=n_{m} \text { and } m \in\{\mu(w \cdot x), \mu(w \cdot x)-1\} .\end{cases}
$$

Finally, no $\gamma^{p}$ not mentioned in the above is allowed in $\mathcal{G}(x)$. 
This concludes the definition of $x \mapsto \mathcal{G}(x)$.

It is straightforward to check that Properties (1)-(6) hold and therefore

$$
\forall x \in 2^{\mathbf{F}_{2}} \forall w \in \mathbf{F}_{2}\left(H(w \cdot x)=\widetilde{w} H(x) \widetilde{w}^{-1}\right) .
$$

The following claim, whose proof is left to the reader, is the analogue of 3.4.

Claim 4.3. Suppose $g_{i}$ is an unbounded quasi-shift satisfying Case 2, i.e.,

$\forall w \in \mathbf{F}_{2} \forall n \in \mathbb{N} \exists z \in \mathbf{F}_{2} \exists u \in \mathbf{F}_{\omega}\left(g_{i}^{-1} \cdot \alpha\left(w, n, l_{i}\right) \cdot g_{i}=u \cdot \alpha\left(z, n, l_{i}\right)^{ \pm 1} \cdot u^{-1}\right)$. Suppose also $\left(w_{m}\right)_{m},\left(n_{m}\right)_{m}$, and $\left(v_{m}\right)_{m}$ witness that $g_{i}$ is bad. Then for all $x \in 2^{\mathbf{F}_{2}}$,

$$
\mu(x)=\infty \Leftrightarrow \forall m\left(\alpha\left(1_{\mathbf{F}_{2}}, n_{m}, l_{i}\right)^{p_{0}} \in \mathcal{G}(x) \vee \alpha\left(1_{\mathbf{F}_{2}}, n_{m}, l_{i}\right)^{p_{1}} \in \mathcal{G}(x)\right)
$$

and if $\mu(x)<\infty$ and $\alpha\left(1_{\mathbf{F}_{2}}, n_{m}, l_{i}\right)^{p} \in \mathcal{G}(x)$ then

$$
p= \begin{cases}p_{2} & \text { if } m \notin\{\mu(x)-1, \mu(x)\}, \\ p_{j} & \text { if } m \in\{\mu(x)-1, \mu(x)\} \text { and } j=J_{\mu(x)}(x)+3 .\end{cases}
$$

We will now show that

$$
\exists i\left(g_{i} H(x) g_{i}^{-1}=H(y) \Rightarrow \exists w \in \mathbf{F}_{2}(y=w \cdot x)\right) .
$$

The reader may find it useful to refer to Figure 2 while checking the details.

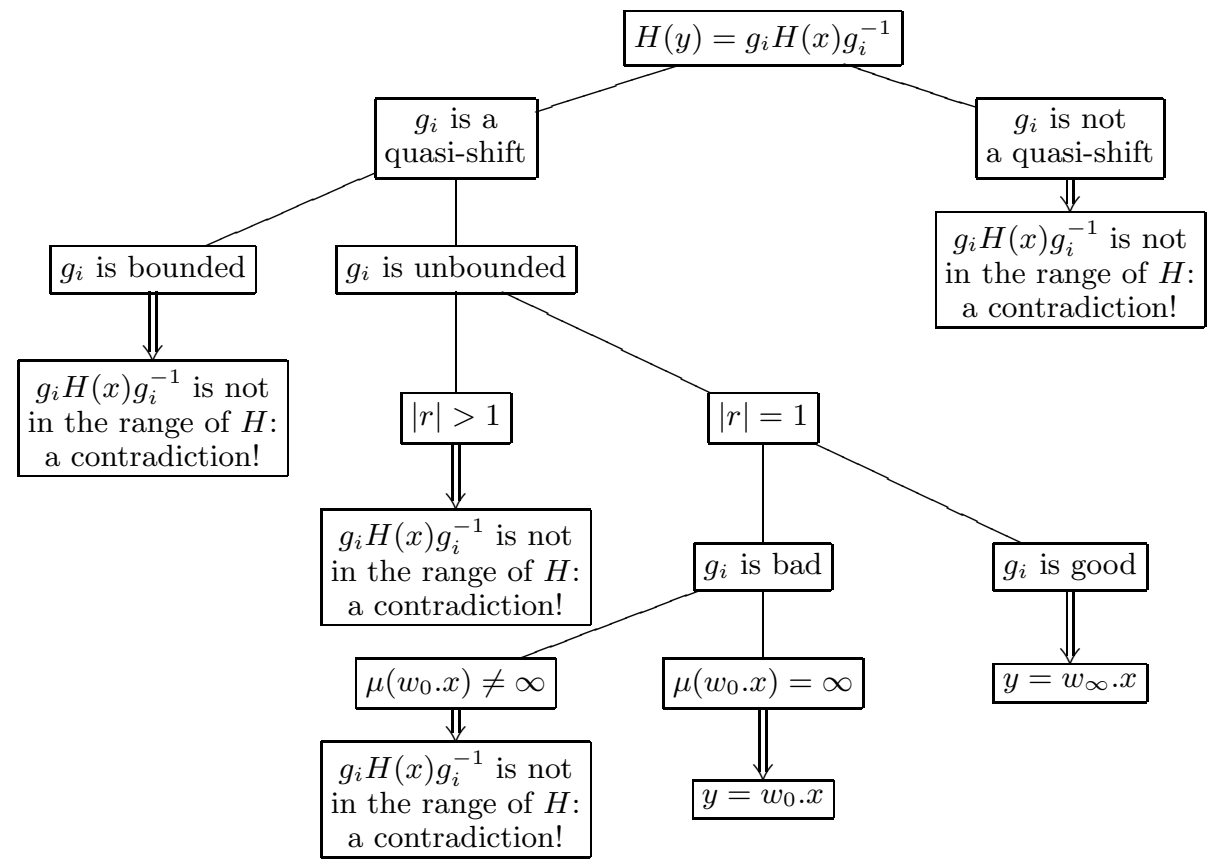

Fig. 2. Plan of the proof of Theorem 1.3 
Assume that

$$
H(y)=g_{i} H(x) g_{i}^{-1} .
$$

Suppose $g_{i}$ is not a quasi-shift, and let $w_{0}, n_{0}, k_{0}, w_{0}^{\prime}, n_{0}^{\prime}, k_{0}^{\prime}$ be as in (i)-(iv).

If (i) holds then we have $k_{0}=l_{i}$ and $\alpha\left(w_{0}, n_{0}, k_{0}\right)^{p} \in \mathcal{G}(y)$, where $p=$ $\min \left(S_{i} \backslash\{r\}\right)$ and $r>0$ is least such that $g_{i}^{-1} \cdot \alpha\left(w_{0}, n_{0}, k_{0}\right)^{r} \cdot g_{i} \in \mathbf{F}_{\omega}$ if such an $r$ exists, or $r=0$ otherwise. Since $\alpha\left(w_{0}, n_{0}, k_{0}\right)^{p} \in H(y)$ we have

$$
g_{i}^{-1} \cdot \alpha\left(w_{0}, n_{0}, k_{0}\right)^{p} \cdot g_{i} \in H(x) \subseteq \mathbf{F}_{\omega},
$$

hence $r>0$. As $r$ and $p$ are relatively prime let $\lambda, \nu \in \mathbb{Z}$ be such that $\lambda r+\nu p=1$ so that

$g_{i}^{-1} \cdot \alpha\left(w_{0}, n_{0}, k_{0}\right) \cdot g_{i}=\left(g_{i}^{-1} \cdot \alpha\left(w_{0}, n_{0}, k_{0}\right)^{r} \cdot g_{i}\right)^{\lambda} \cdot\left(g_{i}^{-1} \cdot \alpha\left(w_{0}, n_{0}, k_{0}\right)^{p} \cdot g_{i}\right)^{\nu} \in \mathbf{F}_{\omega}$ contradicting our assumption case.

If (ii) holds then $l_{i-1}+1=k_{0}<l_{i}=k_{0}^{\prime}, \alpha\left(w_{0}, n_{0}, k_{0}\right)^{p} \in \mathcal{G}(y)$, where $p=\min \left(S_{i}\right)$, and

$$
\forall w, n, r\left(\alpha\left(w, n, k_{0}^{\prime}\right)^{r} \notin \mathcal{G}(x)\right) .
$$

By case hypothesis $\alpha\left(w_{0}^{\prime}, n_{0}^{\prime}, k_{0}^{\prime}\right)$ is a generator in $g_{i}^{-1} \cdot \alpha\left(w_{0}, n_{0}, k_{0}\right)^{p} \cdot g_{i} \in$ $H(x)$, hence by (5) above, $\alpha\left(w_{0}^{\prime}, n_{0}^{\prime}, k_{0}^{\prime}\right)^{q} \in \mathcal{G}(x)$ for some $q \in S_{i}$ such that $q \mid p$. A contradiction.

If (iii) holds then $k_{0}=l_{i}, \alpha\left(w_{0}, n_{0}, l_{i}\right)^{\min \left(S_{i}\right)} \in \mathcal{G}(y)$, and $\alpha\left(w_{0}^{\prime}, n_{0}^{\prime}, l_{i}\right)^{r} \notin$ $\mathcal{G}(x)$, for any $r$. But $g_{i}^{-1} \cdot \alpha\left(w_{0}, n_{0}, l_{i}\right)^{\min \left(S_{i}\right)} \cdot g_{i} \in H(x)$ implies by (5) that $\alpha\left(w_{0}^{\prime}, n_{0}^{\prime}, l_{i}\right)^{q} \in \mathcal{G}(x)$ for some appropriate $q \in S_{i}$. A contradiction.

So suppose (iv) holds. Then $k_{0}=l_{i}, \alpha\left(w_{0}, n_{0}, l_{i}\right)^{p} \in \mathcal{G}(y) \subseteq H(y)$, where $p=\min \left(S_{i}\right)$, and all generators of $g_{i}^{-1} \cdot \alpha\left(w_{0}, n_{0}, l_{i}\right) \cdot g_{i}$ are of level $<l_{i}$. For notational ease let $v=g_{i}^{-1} \cdot \alpha\left(w_{0}, n_{0}, l_{i}\right) \cdot g_{i}$ so that $v^{p} \in H(x)$. If we show that $v \in H(x)$ then we will reach the desired contradiction, since this would imply that $\alpha\left(w_{0}, n_{0}, l_{i}\right)=g_{i} \cdot v \cdot g_{i}^{-1} \in H(y)$, against (5).

If $v$ were a generator then, by case assumption, its level would be between $l_{j-1}$ and $l_{j}$, with $j<i$, so by $v^{p} \in H(x)$ and by (5), there should be a $q \in S_{j}$ such that $q \mid p$, which is absurd. Therefore we may assume that $v \notin \Gamma$. If $v \ldots v$ is in reduced form (in other words: if $v$ starts and ends with different generators) then we are done by (6), so we may assume otherwise. Let $v_{0}$ be the longest initial segment of the word $v \in \mathbf{F}_{\omega}$ such that $v$ ends with $v_{0}^{-1}$, and let $v=v_{0} \cdot v_{1} \cdot v_{0}^{-1}$. Suppose first $v_{0}$ is non-empty. Then either

(a) $v_{0} \cdot v_{1} \cdot v_{0}^{-1}$ is a reduced word, or else

(b) $v_{0}$ ends with the same generator that $v_{1}$ starts with, or else

(c) $v_{1}$ ends with the same generator that $v_{0}^{-1}$ starts with, and (b) fails. If $v_{0}$ is empty, then

(d) $v=\gamma^{h} \cdot u \cdot \gamma^{k}$ with $\gamma \in \Gamma, h, k \in \mathbb{Z} \backslash\{0\}, h+k \neq 0$, and $u$ does not start or end with $\gamma$. 
Note that cases (a), (b), (c), and (d) are mutually exclusive.

Let us deal with case (d) first. Then

$$
v^{p}=\gamma^{h} \cdot u \cdot \gamma^{h+k} \cdot u \ldots u \cdot \gamma^{h+k} \cdot u \cdot \gamma^{k}
$$

is a reduced word, so by (6), $\gamma^{h}, u, \gamma^{k} \in H(x)$ and therefore $v=\gamma^{h} \cdot u \cdot \gamma^{k} \in$ $H(x)$.

If case (a) holds then we have two subcases. Either $v_{1} \ldots v_{1}$ is in reduced form so that $v^{p}$ can be written in reduced form as

$$
v^{p}=v_{0} \cdot \underbrace{v_{1} \ldots v_{1}}_{p} \cdot v_{0}^{-1}
$$

and hence by (6), $v_{0}, v_{1} \in H(x)$, and thus $v \in H(x)$, or else $v_{1}$ starts and ends with the same generator, so by (6) and case (d) applied to $v_{1}$,

$$
v_{0} \cdot v_{1}^{p} \cdot v_{0}^{-1} \in H(x) \Rightarrow v_{0}, v_{1}^{p} \in H(x) \Rightarrow v_{0}, v_{1} \in H(x),
$$

hence $v \in H(x)$.

If case (b) holds, let $\gamma \in \Gamma$ be such that $v_{0}=z \cdot \gamma^{h}$ and $v_{1}=\gamma^{k} \cdot u$ are both reduced words. By case assumption, $z$ does not end with $\gamma$, and $h, k \in \mathbb{Z} \backslash\{0\}$ have the same sign. Then

$$
v^{p}=z \cdot \gamma^{h+k} \cdot u \cdot \gamma^{k} \cdot u \ldots \gamma^{k} \cdot u \cdot \gamma^{-h} \cdot z^{-1}
$$

is in reduced form, so by (6), $z, u, \gamma^{k}, \gamma^{-h} \in H(x)$ and therefore

$$
v=\left(z \cdot \gamma^{h}\right) \cdot\left(\gamma^{k} \cdot u\right) \cdot\left(z \cdot \gamma^{h}\right)^{-1} \in H(x) .
$$

Case (c) is similar and left to the reader.

Therefore in all cases $v \in H(x)$, reaching a contradiction. Thus

$g_{i}$ is not a quasi-shift above $l_{i-1} \Rightarrow g_{i} H(x) g_{i}^{-1}$ is not in the range of $H$.

So we may assume from this point on that $g_{i}$ is a quasi-shift above $l_{i-1}$.

Suppose $g_{i}$ is bounded and let $\alpha\left(w_{0}, n_{0}, l_{i}\right)$ witness this. Let also $\alpha\left(z, n_{0}, l_{i}\right)$ be a generator in $v=g_{i}^{-1} \cdot \alpha\left(w_{0}, n_{0}, l_{i}\right) \cdot g_{i}$. Since $\alpha\left(w_{0}, n_{0}, l_{i}\right)^{p} \in$ $H(y)$, we have $g_{i}^{-1} \cdot \alpha\left(w_{0}, n_{0}, l_{i}\right)^{p} \cdot g_{i}=v^{p} \in H(x)$, hence its generators to some appropriate prime powers must be in $\mathcal{G}(x)$. In particular, this must be true of $\alpha\left(z, n_{0}, l_{i}\right)$ : by properties (1) and (5) there are $q \in S_{i}$ and $k$ such that $\alpha\left(z, n_{0}, l_{i}\right)^{k}$ occurs in $v^{p}, q \mid k$ and $\alpha\left(z, n_{0}, l_{i}\right)^{q} \in \mathcal{G}(x)$. But by definition of $\mathcal{G}$, if $g_{i}$ is bounded then $q=p>|k|$, a contradiction.

Thus

$g_{i}$ is bounded $\Rightarrow g_{i} H(x) g_{i}^{-1}$ is not in the range of $H$.

So we may assume that $g_{i}$ is unbounded.

Suppose Case 1 holds: let $g_{i}^{-1} \cdot \alpha\left(w_{0}, n_{0}, l_{i}\right) \cdot g_{i}=v=v_{0} \cdot \alpha\left(z, n_{0}, l_{i}\right)^{r}$. $v_{0}^{-1}$ and $r=r\left(w_{0}, n_{0}\right),|r|>1$. Since $\alpha\left(w_{0}, n_{0}, l_{i}\right)^{p} \in H(y)$ and $v^{p}=$ 
$v_{0} \cdot \alpha\left(z, n_{0}, l_{i}\right)^{r p} \cdot v_{0}^{-1} \in H(x)$ is reduced, we have $v_{0}, \alpha\left(z, n_{0}, l_{i}\right)^{p} \in H(x)$ by properties (5) and (6). Therefore $v_{0} \cdot \alpha\left(z, n_{0}, l_{i}\right)^{p} \cdot v_{0}^{-1} \in H(x)$ and computing in $H(y)$, we get

$$
\begin{aligned}
\left(g_{i} \cdot v_{0} \cdot \alpha\left(z, n_{0}, l_{i}\right)^{p} \cdot v_{0}^{-1} \cdot g_{i}^{-1}\right)^{r} & =g_{i} \cdot v_{0} \cdot \alpha\left(z, n_{0}, l_{i}\right)^{p r} \cdot v_{0}^{-1} \cdot g_{i}^{-1} \\
& =g_{i} \cdot v^{p} \cdot g_{i}^{-1}=\alpha\left(w_{0}, n_{0}, l_{i}\right)^{p}
\end{aligned}
$$

a contradiction, since $\alpha\left(w_{0}, n_{0}, l_{i}\right)^{p} \in \mathcal{G}(y)$ is a generator of $H(y)$ and hence it is not the $r$ th power of any element.

Thus

$g_{i}$ is unbounded $\&|r|>1 \Rightarrow g_{i} H(x) g_{i}^{-1}$ is not in the range of $H$. $=1)$.

Therefore we may assume we are in Case 2, that is, $\forall w \in \mathbf{F}_{2} \forall n(|r(w, n)|$

Suppose $g_{i}$ is good, so that

$$
g_{i}^{-1} \cdot \alpha\left(1_{\mathbf{F}_{2}}, n_{m}, l_{i}\right) \cdot g_{i}=v_{m} \cdot \alpha\left(w_{\infty}, n_{m}, l_{i}\right)^{r\left(1_{\mathbf{F}_{2}}, n_{m}\right)} \cdot v_{m}^{-1} .
$$

Then, by property (6), for $e=0,1$,

$$
\begin{aligned}
y\left(u_{m}\right)=e & \Leftrightarrow \alpha\left(1_{\mathbf{F}_{2}}, n_{m}, l_{i}\right)^{p_{e}} \in H(y) \\
& \Leftrightarrow v_{m} \cdot \alpha\left(w_{\infty}, n_{m}, l_{i}\right)^{p_{e} \cdot r\left(1_{\mathbf{F}_{2}}, n_{m}\right)} \cdot v_{m}^{-1} \in H(x) \\
& \Rightarrow \alpha\left(w_{\infty}, n_{m}, l_{i}\right)^{p_{e}} \in H(x) \\
& \Leftrightarrow w_{\infty} \cdot x\left(u_{m}\right)=e
\end{aligned}
$$

that is, $w_{\infty} \cdot x=y$. Thus

$$
g_{i} \text { is good } \Rightarrow y=w_{\infty} . x
$$

Therefore we may assume $g_{i}$ is bad, hence $g_{i}^{-1} \cdot \alpha\left(1_{\mathbf{F}_{2}}, n_{m}, l_{i}\right) \cdot g_{i}=$ $v_{m} \cdot \alpha\left(w_{m}, n_{m}, l_{i}\right)^{r\left(1_{\mathbf{F}_{2}}, n_{m}\right)} \cdot v_{m}^{-1}$. Suppose $\mu\left(w_{0} \cdot x\right)=\infty$. Then by Claim 3.2 in the proof of $3.1, \forall m\left(w_{m} . x=w_{0} . x\right)$ hence

$$
\forall m\left(\mu\left(w_{m} \cdot x\right)=\infty \text { and } H\left(w_{m} \cdot x\right)=H\left(w_{0} \cdot x\right)\right) .
$$

For $p \in S_{i}$, using property (6) and the fact that $r\left(1_{\mathbf{F}_{2}}, n_{m}\right)= \pm 1$ we get

$$
\begin{aligned}
\alpha\left(1_{\mathbf{F}_{2}}, n_{m}, l_{i}\right)^{p} \in H(y) & \Leftrightarrow v_{m} \cdot \alpha\left(w_{m}, n_{m}, l_{i}\right)^{p \cdot r\left(1_{\mathbf{F}_{2}}, n_{m}\right)} \cdot v_{m}^{-1} \in H(x) \\
& \Rightarrow \alpha\left(w_{m}, n_{m}, l_{i}\right)^{p} \in H(x) \\
& \Leftrightarrow \alpha\left(1_{\mathbf{F}_{2}}, n_{m}, l_{i}\right)^{p} \in H\left(w_{m} \cdot x\right)=H\left(w_{0} \cdot x\right) \\
& \Rightarrow p=p_{0} \vee p=p_{1} .
\end{aligned}
$$


By Claim 4.3, $\mu(y)=\infty$ and hence, for $e=0,1$,

$$
\begin{aligned}
y\left(u_{m}\right)=e & \Leftrightarrow \alpha\left(1_{\mathbf{F}_{2}}, n_{m}, l_{i}\right)^{p_{e}} \in H(y) \\
& \Rightarrow \alpha\left(1_{\mathbf{F}_{2}}, n_{m}, l_{i}\right)^{p_{e}} \in H\left(w_{0} \cdot x\right) \\
& \Leftrightarrow w_{0} \cdot x\left(u_{m}\right)=e,
\end{aligned}
$$

that is, $w_{0} \cdot x=y$. Thus

$$
g_{i} \text { is } \operatorname{bad} \& \mu\left(w_{0} \cdot x\right)=\infty \Rightarrow w_{0} \cdot x=y .
$$

Suppose $\mu\left(w_{0} \cdot x\right)=m<\infty$. Then by 3.2 ,

$$
\forall j<m\left(\mu\left(w_{j} . x\right)=m \& J_{m}\left(w_{j} \cdot x\right)=J_{m}\left(w_{0} \cdot x\right)\right) \text { and } \mu\left(w_{m} . x\right) \leq m .
$$

Let $e_{j} \in\{0, \ldots, 5\}$ be the unique value such that

$$
\alpha\left(w_{j}, n_{j}, l_{i}\right)^{p_{e_{j}} \in \mathcal{G}(x) .}
$$

Then the first $m$ elements of $\left(e_{j}\right)_{j}$ are $\left(2, \ldots, 2, J_{m}\left(w_{0} . x\right)+3\right)$ and by Claim 4.3 the next element must be $J_{m}\left(w_{0} \cdot x\right)+3$.

Suppose $\mu\left(w_{m} \cdot x\right)=m$. Since $w_{0} \cdot x=w_{m-1} \cdot x$ and $z_{m} \cdot w_{0} \cdot x=w_{m} \cdot x$ are distinct, $J_{m}\left(w_{0} \cdot x\right) \neq J_{m}\left(w_{m} \cdot x\right)$, a contradiction.

Suppose $\mu\left(w_{m} . x\right)<m$. Then $e_{m}=2 \neq J_{m}\left(w_{0} \cdot x\right)+3$, a contradiction again.

Thus

$g_{i}$ is bad \& $\mu\left(w_{0} \cdot x\right)<\infty \Rightarrow g_{i} H(x) g_{i}^{-1}$ is not in the range of $H$.

This concludes the proof.

\section{References}

[1] R. Dougherty, S. Jackson and A. S. Kechris, The structure of hyperfinite Borel equivalence relations, Trans. Amer. Math. Soc. 341 (1994), 193-225.

[2] R. Dougherty and A. S. Kechris, How many Turing degrees are there?, in: Computability Theory and its Applications (Boulder, CO, 1999), Contemp. Math. 257, Amer. Math. Soc., 2000, 83-94.

[3] S. Gao, Coding subset shift by subgroup conjugacy, Bull. London Math. Soc. 32 (2000), 653-657.

[4] G. Hjorth and A. S. Kechris, Borel equivalence relations and classifications of countable models, Ann. Pure Appl. Logic 82 (1996), 221-272.

[5] A. S. Kechris, Classical Descriptive Set Theory, Grad. Texts in Math. 156, Springer, 1995.

[6] A. S. Kechris and Y. N. Moschovakis (eds.), Cabal Seminar 76-77, Lecture Notes in Math. 689, Springer, 1978.

[7] A. S. Kechris, S. Solecki and S. Todorčević, Borel chromatic numbers, Adv. Math. 141 (1999), 1-44. 
[8] G. Stuck and R. J. Zimmer, Stabilizers for ergodic actions of higher rank semisimple groups, Ann. of Math. 139 (1994), 723-747.

[9] S. Thomas and B. Veličković, On the complexity of the isomorphism relation for finitely generated groups, J. Algebra 217 (1999), 352-373.

[10] S. Wagon, The Banach-Tarski Paradox, Encyclopedia Math. Appl. 24, Cambridge Univ. Press, 1985.

Dipartimento di Matematica

Università di Torino

via Carlo Alberto 10

10123 Torino, Italy

E-mail: andretta@dm.unito.it

camerlo@dm.unito.it
Department of Mathematics University of California at Los Angeles Los Angeles, CA 900095-1555, U.S.A. E-mail: greg@math.ucla.edu

Received 20 September 1999;

in revised form 12 August 2000 